\title{
The Voronoi Diagram of Three Lines
}

\author{
Hazel Everett • Daniel Lazard • Sylvain Lazard • \\ Mohab Safey El Din
}

Received: 5 September 2007 / Revised: 23 February 2009 / Accepted: 23 February 2009 /

Published online: 28 April 2009

(C) Springer Science+Business Media, LLC 2009

\begin{abstract}
We give a complete description of the Voronoi diagram, in $\mathbb{R}^{3}$, of three lines in general position, that is, that are pairwise skew and not all parallel to a common plane. In particular, we show that the topology of the Voronoi diagram is invariant for three such lines. The trisector consists of four unbounded branches of either a nonsingular quartic or of a nonsingular cubic and a line that do not intersect in real space. Each cell of dimension two consists of two connected components on a hyperbolic paraboloid that are bounded, respectively, by three and one of the branches of the trisector. We introduce a proof technique which relies heavily upon modern tools of computer algebra and is of interest in its own right.

This characterization yields some fundamental properties of the Voronoi diagram of three lines. In particular, we present linear semi-algebraic tests for separating the two connected components of each two-dimensional Voronoi cell and for separating the four connected components of the trisector. This enables us to answer queries of the form, given a point, determine in which connected component of which cell it lies. We also show that the arcs of the trisector are monotonic in some direction. These properties imply that points on the trisector of three lines can be sorted along each branch using only linear semi-algebraic tests.
\end{abstract}

A preliminary version of this paper appeared in 2007 in the Proceedings of the 23rd Annual ACM Symposium on Computational Geometry [18].

H. Everett · S. Lazard $(\bowtie)$

LORIA, INRIA Lorraine, University Nancy 2, Nancy, France

e-mail: Sylvain.Lazard@loria.fr

H. Everett

e-mail: Hazel.Everett@loria.fr

D. Lazard · M. Safey El Din

LIP6, INRIA Rocquencourt, University Pierre et Marie Curie, Paris, France

M. Safey El Din

e-mail: Mohab.Safey@lip6.fr 
Keywords Computational geometry $\cdot$ Computer algebra $\cdot$ Voronoi diagram $\cdot$ Medial axis · Quadric surface intersection

\section{Introduction}

The Voronoi diagram of a set of disjoint objects is a decomposition of the space into cells, one cell per object, such that the cell associated with an object consists of all points that are closer to that object than to any other object. In this paper, we consider the Voronoi diagram of lines in $\mathbb{R}^{3}$ under the Euclidean metric.

Voronoi diagrams have been the subject of a tremendous amount of research. For points under the Euclidean metric, these diagrams and their complexities are well understood, and optimal algorithms and robust efficient implementations exist for computing them in any dimension (see, for instance, [2, 4, 20, 35]). Such diagrams, whose bisectors are hyperplanes, are called affine diagrams.

Nonaffine Voronoi diagrams, whose bisectors are curved, are far less well understood. Voronoi diagrams of points in various dimensions but with additively and multiplicatively weighted metrics, such as Möbius, Apollonius (or Johnson-Mehl), or anisotropic diagrams, are typical examples (see [5] for a recent survey). Voronoi diagrams of segments [22] or ellipses [16] in two dimensions are other examples of nonaffine diagrams.

Voronoi diagrams of lines, segments, and polyhedra in three dimensions are nonaffine diagrams for which very little is known. In particular, determining the combinatorial complexity of the Voronoi diagram of $n$ lines or line segments in $\mathbb{R}^{3}$ is an outstanding open problem. The best known lower bound is $\Omega\left(n^{2}\right)$, and the best upper bound is $O\left(n^{3+\epsilon}\right)$ [47]. It is conjectured that the complexity of such diagrams is near-quadratic. In the restricted case of a set of $n$ lines with a fixed number, $c$, of possible orientations, Koltun and Sharir have shown an upper bound of $O\left(n^{2+\epsilon}\right)$ for any $\epsilon>0$ [24].

There are few algorithms for computing exactly the Voronoi diagram of linear objects. Most of this work has been done in the context of computing the medial axis of a polyhedron, i.e., the Voronoi diagram of the faces of the polyhedron [11, 32]. Recently, some progress has been made on the related problem of computing arrangements of quadrics (each cell of the Voronoi diagram is a cell of such an arrangement) [3, 13-15, 23, 33, 43, 45]. Finally, there have been many papers reporting algorithms for computing approximations of the Voronoi diagram (see, for instance, $[12,17,21,48])$.

In this paper, we address the fundamental problem of understanding the structure of the Voronoi diagram of three lines. A robust and effective implementation of Voronoi diagrams of three-dimensional linear objects requires a complete and thorough treatment of the base cases, that is, the diagrams of three and four lines, points, or planes. We also believe that this is required in order to make progress on complexity issues and in particular for proving tight worst-case bounds. We provide here a full and complete characterization of the geometry and topology of the elementary though difficult case of the Voronoi diagram of three lines in general position.

Main Results Our main result, which settles a conjecture of Koltun and Sharir [24], is the following (see Fig. 1). 


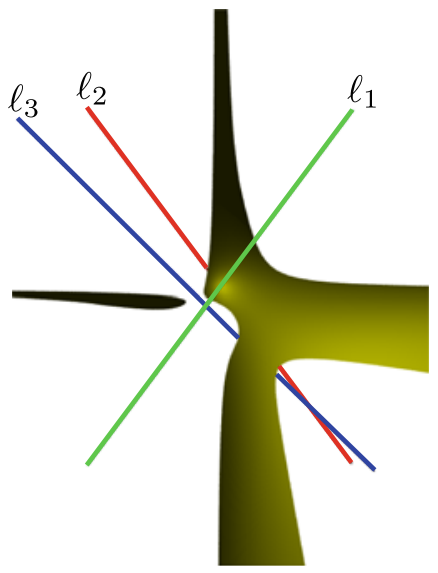

(a)

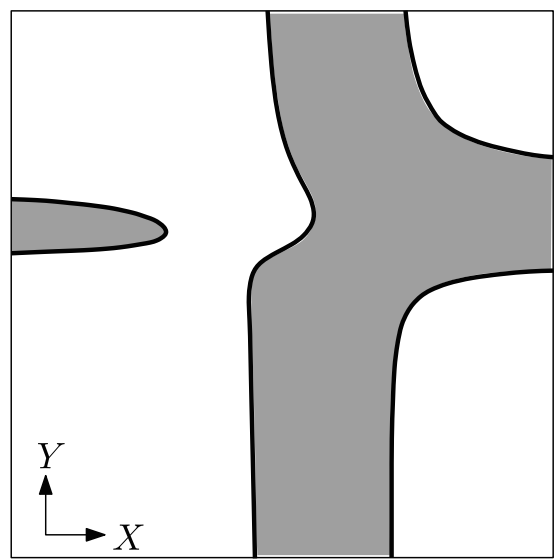

(b)

Fig. 1 Voronoi diagram of 3 lines $\ell_{1}, \ell_{2}$, and $\ell_{3}$ in general position: (a) Voronoi $2 \mathrm{D}$ face of $\ell_{1}$ and $\ell_{2}$, i.e., set of points equidistant to $\ell_{1}$ and $\ell_{2}$ and closer to them than to $\ell_{3}$. (b) Orthogonal projection of a 2D face on a plane $\mathcal{P}$ with coordinate system $(X, Y)$; the plane's normal is parallel to the common perpendicular of $\ell_{1}$ and $\ell_{2}$, and the $X$ - and $Y$-axes are parallel to the two bisector lines (in $\mathcal{P}$ ) of the projection of $\ell_{1}$ and $\ell_{2}$ on $\mathcal{P}$. The $2 \mathrm{D}$ face is bounded by four branches of a nonsingular quartic

Theorem 1 The topology of the Voronoi diagram of three pairwise skew lines that are not all parallel to a common plane is invariant. The trisector consists of four infinite branches of either a nonsingular quartic ${ }^{1}$ or a nonsingular cubic and a line that do not intersect in $\mathbb{P}^{3}(\mathbb{R})$. Each cell of dimension two consists of two connected components on a hyperbolic paraboloid that are bounded, respectively, by three and one of the branches of the trisector.

We introduce, for the proof of Theorem 1, a new proof technique which relies heavily upon modern tools of computer algebra and which is of interest in its own right. We also provide a geometric characterization of the configurations of three lines in general position whose trisector is not generic, that is, consists of a cubic and a line.

Theorem 2 The trisector of three pairwise skew lines that are not all parallel to a common plane consists of a cubic and a line if and only if the hyperboloid of one sheet containing the three skew lines is of revolution.

\footnotetext{
${ }^{1}$ By a nonsingular quartic we mean an irreducible curve of degree four with no singular point in $\mathbb{P}^{3}(\mathbb{C})$. Recall that a point $p \in \mathbb{P}^{3}(\mathbb{C})$ of a surface $S$ is said to be singular if its tangent plane is not defined at $p$, that is, all partial derivatives of the square-free polynomial defining $S$ are zero at $p$. Similarly, a point $p \in \mathbb{P}^{3}(\mathbb{C})$ of a curve $C$ defined by the two implicit equations $E_{1}=E_{2}=0$ is singular if the rank of the Jacobian matrix of $C$ (the matrix of partial derivatives of $E_{1}$ and $E_{2}$ ) is at most 1 when evaluated at $p$. (Note that the ideal generated by $E 1$ and $E 2$ should contain all the polynomials vanishing on $C$.) A curve is said to be singular in $\mathbb{P}^{3}(\mathbb{C})$, or simply singular, if it contains at least a singular point in the complex projective space $\mathbb{P}^{3}(\mathbb{C})$. A curve is said to be singular in $\mathbb{P}^{3}(\mathbb{R})$ if it contains at least a singular point in the real projective space $\mathbb{P}^{3}(\mathbb{R})$.
} 
This work enables us to prove some fundamental properties of the Voronoi diagram of three lines which are likely to be critical for the analysis of the complexity and the development of efficient algorithms for computing Voronoi diagrams and medial axes of lines or polyhedra. In particular, we obtain the following results.

Monotonicity property Given three pairwise skew lines that are not all parallel to a common plane, there is a direction in which all four branches of the trisector are monotonic.

Theorem 3 Given a point $p$ that lies on a two-dimensional cell of the Voronoi diagram of three pairwise skew lines that are not all parallel to a common plane, deciding on which connected component of the cell point p lies can be done by evaluating the sign of linear forms in the coordinates of $p$; similarly, if $p$ lies on the trisector. Furthermore, points on any one branch of the trisector may be ordered by comparing the values of a linear form in the coordinates of the points. Moreover, if the three input lines have rational coefficients, the coefficients of these linear forms may be chosen rational.

Notice that these tests enable us to answer queries of the form, given a point, determine in which connected component of which cell it lies. Notice also that these tests should be useful for computing the Voronoi diagram of $n$ lines since computing the vertices of such diagrams requires locating the points equidistant to four lines on a Voronoi arc of three of these lines.

Before describing the organization of the paper and of the proofs of our main results, we briefly recall some related work at the frontier of computer algebra and computational geometry and position our work in that context.

There is a large body of work that deals with automated Geometry Theorem Proving, which consists in finding mechanical means of proving elementary geometry theorems (see, for instance, [8]). Another topic, closer to our concerns, deals with the applications of computer algebra to computational geometry; examples can be found in problems relating to motion planning (see, e.g., [26, 44]) or to computing the topology of algebraic curves (see [7] for a recent reference). Our work is, however, more closely related to computer-assisted proofs such as the proofs of the four color theorem [1, 37] or the NP-hardness of minimum-weight triangulation [34]. However, in this paper, we are interested in proving properties on algebraic varieties of relatively high degree and there exists, up to our knowledge, very few computer-aided proofs for obtaining such results (see [28, 29] for two such results in geometry).

The rest of the paper is organized as follows. We first characterize, in Sect. 2, the trisector of three lines in general position, We then present, in Sect. 3, some fundamental properties of the Voronoi diagram of three lines and prove the Monotonicity Property (Proposition 18). We then complete the proof of Theorem 1 in Sect. 4 and give a simple proof of Theorem 2 in Sect. 5. Finally, in Sect. 6, we present algorithms for separating the components of each cell of the Voronoi diagram and prove Theorem 3.

\section{Structure of the Trisector}

We consider three lines in general position, that is, pairwise skew and not all parallel to the same plane. The idea of the proof of Theorem 1 is to prove that the topology 
of the trisector is invariant by continuous deformation on the set of all triplets of three lines in general position which consists of two connected components. The result will then follow from the analysis of any pair of examples, one from each component.

To prove that the topology of the trisector is invariant by continuous deformation on the set of all triplets of three lines in general position, we first show, in this section, that the trisector of three lines in general position is always homeomorphic to four lines that do not pairwise intersect. To prove this, we show that the trisector is always nonsingular in $\mathbb{P}^{3}(\mathbb{R})$ and has four simple real points at infinity. To show that the trisector is always nonsingular in $\mathbb{P}^{3}(\mathbb{R})$, we study the type of the intersection of two bisectors, which are hyperbolic paraboloids.

We use the classical result that the intersection of two quadrics is a nonsingular quartic (in $\mathbb{P}^{3}(\mathbb{C})$ ) unless the characteristic equation of their pencil has (at least) a multiple root. In order to determine when this equation has a multiple root, we determine when its discriminant $\Delta$ is zero.

This discriminant has several factors, some of which are trivially always positive. We prove that the remaining, so-called "gros facteur," is zero (over the reals) only if a simple polynomial $F$ is zero (see the Main Lemma in Sect. 2.2). We provide two proofs of this result. We first give a short direct proof in Sect. 2.2.1. Although this proof is elegant, it provides no insight into how we discovered the result. We also present a second proof in Sect. 2.2.2, which relies heavily upon sophisticated tools of modern algebra and does not require any detailed understanding of the geometry of the problem. This longer proof is indeed how we originally obtained Theorems 1 and 2, and only with the geometric insight gained from this process were we able to find the shorter proof. We believe this longer proof to be of interest in its own right because it demonstrates a technique which could be applied to other problems.

This proof goes as follows. We first show, in Lemma 5, that the gros facteur is never negative using the RAGLiB Maple package [40]. This implies that it zero only when all its partial derivatives are zero (Lemma 6). We thus consider, in Sect. 2.3, the system that consists of the gros facteur and all its partial derivatives, and compute its Gröbner basis [10]. Recall that the Gröbner basis of a system of polynomials is a particular set of polynomials that has the same set of roots as the initial system (more precisely, it generates the same ideal). It also has the property that it consists of exactly one polynomial equal to 1 if and only if the initial system has no complex root.

The Gröbner basis we compute consists of three polynomials of degree six. We first determine the aforementioned polynomial $F$ by factoring these polynomials. We then consider separately two components of solutions, one for which $F$ is zero, and the other for which $F \neq 0$. When $F \neq 0$, some manipulations and simplifications, which are interesting in their own right, yield another Gröbner basis, with the same real roots, which consists of three equations of degree four. We show that one of these equations has no real root, which implies that the system has no real root and thus that the gros facteur is strictly positive on the considered component. We can thus conclude that $\Delta=0$ only if $F=0$ and thus that, when $F \neq 0$, the trisector is always a nonsingular quartic in $\mathbb{P}^{3}(\mathbb{R})$. 
Fig. 2 Three lines in general position

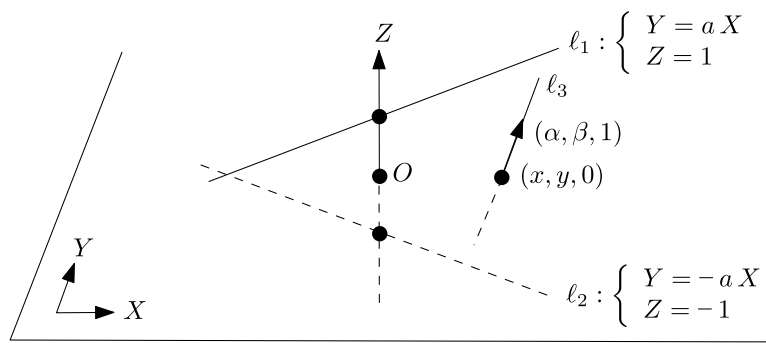

Then, when the polynomial $F=0$, we show in Sect. 2.4, by substituting $F=0$ into $\Delta$ and by using the classification of the intersection of quadrics over the reals [14], that the trisector is a nonsingular cubic and a line that do not intersect in $\mathbb{P}^{3}(\mathbb{R})$.

We can thus conclude that the trisector is always a nonsingular quartic or a nonsingular cubic and a line that do not intersect in real space and thus that the trisector is always nonsingular in $\mathbb{P}^{3}(\mathbb{R})$. We then prove that the trisector always contains four simple real points at infinity and thus that it is always homeomorphic to four lines that do not pairwise intersect.

\subsection{Preliminaries}

Let $\ell_{1}, \ell_{2}$, and $\ell_{3}$ be three lines in general position, i.e., that are pairwise skew and not all parallel to a common plane. Refer to Fig. 2. Let $(X, Y, Z)$ denote a Cartesian coordinate system. Without loss of generality, we assume that $\ell_{1}$ and $\ell_{2}$ are both parallel to $X Y$-plane, pass through $(0,0,1)$ and $(0,0,-1)$, respectively, and have directions that are symmetric with respect to the $X Z$-plane. More precisely, we assume that the line $\ell_{1}$ is defined by point $p_{1}=(0,0,1)$ and vector $v_{1}=(1, a, 0)$, and the line $\ell_{2}$ is defined by the point $p_{2}=(0,0,-1)$ and vector $v_{2}=(1,-a, 0), a \in \mathbb{R}$. Moreover, since the three lines are not all parallel to a common plane, $\ell_{3}$ is not parallel to the plane $z=0$, and so we can assume that the line $\ell_{3}$ is defined by point $p_{3}=(x, y, 0)$ and vector $v_{3}=(\alpha, \beta, 1), x, y, \alpha, \beta \in \mathbb{R}$.

We denote by $\mathcal{H}_{i, j}$ the bisector of lines $\ell_{i}$ and $\ell_{j}$ and by $V_{i j}$ the Voronoi cell of lines $\ell_{i}$ and $\ell_{j}$, i.e., the set of points equidistant to $\ell_{i}$ and $\ell_{j}$ and closer to them than to $\ell_{k}, k \neq i, j$. We recall that the three-dimensional Voronoi cells are connected and that the bisector of two skew lines is a right hyperbolic paraboloid, that is, has equation of the form $Z=\gamma X Y, \gamma \in \mathbb{R}^{\star}$, in some orthogonal coordinate system (see, for instance, [24]); for completeness, we present a proof of this fact.

Lemma 4 The bisector of two skew lines is a right hyperbolic paraboloid.

Proof The bisector of two lines $\ell_{i}$ and $\ell_{j}$ is the set of points $p$ satisfying the equation

$$
\frac{\left\|\left(p-p_{i}\right) \times v_{i}\right\|^{2}}{\left\|v_{i}\right\|^{2}}=\frac{\left\|\left(p-p_{j}\right) \times v_{j}\right\|^{2}}{\left\|v_{j}\right\|^{2}} .
$$


If suffices to prove the lemma for the two lines $\ell_{1}$ and $\ell_{2}$. For these lines, the above equation simplifies into the following equation of a right hyperbolic paraboloid:

$$
Z=-\frac{a}{1+a^{2}} X Y
$$

The trisector of our three lines is the intersection of two right hyperbolic paraboloids, say $\mathcal{H}_{1,2}$ and $\mathcal{H}_{1,3}$. The intersection of two arbitrary hyperbolic paraboloids may be singular; it may be a nodal or cuspidal quartic, two secant conics, a cubic and a line that intersect, a conic and two lines crossing on the conic, etc. We show here that the trisector is always nonsingular in $\mathbb{P}^{3}(\mathbb{R})$ by studying the characteristic polynomial of the pencil of $\mathcal{H}_{1,2}$ and $\mathcal{H}_{1,3}$.

Let $Q_{1,2}$ and $Q_{1,3}$ be matrix representations of $\mathcal{H}_{1,2}$ and $\mathcal{H}_{1,3}$, i.e., the Hessian of the quadratic form associated with the surface (see, for instance, [13]). The pencil of $Q_{1,2}$ and $Q_{1,3}$ is the set of their linear combinations, that is, $P(\lambda)=\left\{\lambda Q_{1,2}+Q_{1,3}\right.$, $\forall \lambda \in \mathbb{R} \cup\{\infty\}\}$. The characteristic polynomial of the pencil is the determinant, $\mathcal{D}(\lambda)=\operatorname{det}(P(\lambda))$, which is a degree four polynomial in $\lambda$. The intersection of any two quadrics is a nonsingular quartic, in $\mathbb{P}^{3}(\mathbb{C})$, if and only if the characteristic equation of the corresponding pencil does not have any multiple roots (in $\mathbb{C}$ ) [46] (see also [14]). A nonsingular quartic of $\mathbb{P}^{3}(\mathbb{C})$ is, in $\mathbb{P}^{3}(\mathbb{R})$, either empty or a nonsingular quartic. Thus, since the trisector of our three lines cannot be the empty set in $\mathbb{R}^{3}$, the trisector is a smooth quartic in $\mathbb{P}^{3}(\mathbb{R})$ if and only if the characteristic equation of the pencil does not have any multiple roots (in $\mathbb{C}$ ).

The characteristic polynomial of the pencil is fairly complicated (roughly one page in the format of (3)). However, by the change of variable $\lambda \rightarrow 2 \lambda\left(1+\alpha^{2}+\beta^{2}\right)$ and by dividing out the positive factor $\left(1+a^{2}\right)^{2}\left(1+\alpha^{2}+\beta^{2}\right)^{3}$, the polynomial simplifies, without changing its roots, to the following, which we still denote by $\mathcal{D}(\lambda)$ for simplicity:

$$
\begin{aligned}
\mathcal{D}(\lambda)= & \left(\alpha^{2}+\beta^{2}+1\right) a^{2} \lambda^{4}-2 a\left(2 a \beta^{2}+a y \beta+a \alpha x-\beta \alpha+2 a+2 a \alpha^{2}-\beta \alpha a^{2}\right) \lambda^{3} \\
& +\left(\beta^{2}+6 a^{2} \beta^{2}-2 \beta x a^{3}-6 \beta \alpha a^{3}+6 y \beta a^{2}-6 a \beta \alpha-2 a \beta x+6 \alpha x a^{2}+y^{2} a^{2}\right. \\
& \left.-2 a \alpha y+x^{2} a^{2}-2 y \alpha a^{3}+6 a^{2} \alpha^{2}+a^{4} \alpha^{2}+4 a^{2}\right) \lambda^{2} \\
& -2\left(x a-y a^{2}-2 \beta a^{2}-\beta+2 a \alpha+\alpha a^{3}\right)(x a-y-\beta+a \alpha) \lambda+\left(1+a^{2}\right)(x a-y-\beta+a \alpha)^{2} .
\end{aligned}
$$

Let $\Delta$ be the discriminant of the characteristic polynomial $\mathcal{D}(\lambda)$ (with respect to $\lambda$ ). Recall that $\Delta=0$ if and only if $\mathcal{D}(\lambda)$ admits a multiple root, that is, if and only if the trisector is not a smooth quartic. The discriminant $\Delta$, computed with Maple [31], is equal to

$$
16 a^{4}(a x-y-\beta+a \alpha)^{2}(y+a x-a \alpha-\beta)^{2}
$$

times a factor that we refer to as the gros facteur, which is a rather large polynomial of degree 18 in 5 variables with 253 monomials, of which we only show 3 out 
of 33 lines:

$$
\begin{aligned}
\text { gros_facteur }= & 8 a^{8} \alpha^{4} y^{2}+7 a^{4} \beta^{2} x^{4}-4 a \beta^{3} x+16 a^{8} \beta^{4} x^{4}+32 a^{4} \alpha^{2} y^{2}+2 a^{6} \alpha^{2} \beta^{4} x^{2}+38 a^{8} \alpha^{2} x^{2} \\
& +2 y^{4} \beta^{2} a^{4} \alpha^{2}+44 a^{8} \alpha^{2} \beta^{2} x^{2}+\cdots+22 a^{4} y^{2} \beta^{2} x^{2}+y^{6} a^{6}+\alpha^{2} y^{6} a^{6}-2 \beta x \alpha y^{5} a^{6} \\
& +x^{6} a^{6}+10 \beta x^{3} a^{7} \alpha^{2}+2 y \alpha^{3} a^{7} x^{2}-32 a^{3} \alpha^{2} y^{2} \beta x+28 a^{3} \beta^{2} x^{2} \alpha y-24 a^{2} \beta^{3} y \alpha x .
\end{aligned}
$$

In the sequel, all polynomials are considered over the reals, that is, for $\lambda, a, \alpha, \beta$, $x, y$ in $\mathbb{R}$, unless specified otherwise.

All the computations were performed on a PC Intel(R) Xeon(TM) CPU $3.20 \mathrm{GHz}$ with 4 GBytes of RAM.

\subsection{The Main Lemma}

We find in this section simple algebraic constraints that are satisfied when discriminant $\Delta$ is equal to zero. Precisely, we prove the following lemma.

Main Lemma The discriminant $\Delta$ is equal to zero only if $y+a \alpha=0$ or $a x+\beta=0$.

Note that the problem is to prove this lemma and also to obtain these two simple equations, which is a difficult problem since $\Delta$ is a fairly large polynomial. As discussed in the overview of the proof, we first present a short direct proof of the Main Lemma and then present our original proof.

\subsubsection{Direct Proof of the Main Lemma}

Note first that the discriminant $\Delta$ is equal to zero if and only if the gros facteur is zero. Indeed, the polynomial (4) is not equal to zero under our general position assumption: $a=0$ is equivalent to saying that lines $\ell_{1}$ and $\ell_{2}$ are parallel and the two other factors of (4) are equal to the square of $\operatorname{det}\left(p_{i}-p_{3}, v_{i}, v_{3}\right)$ for $i=1,2$ and thus are equal to zero if and only if $\ell_{i}$ and $\ell_{3}$ are coplanar for $i=1,2$.

Now, it can be easily verified (using, for instance, Maple) that the gros facteur is, in fact, the discriminant of the characteristic polynomial of the $3 \times 3$ top-left submatrix of the matrix representation of the quadric containing $\ell_{1}, \ell_{2}$, and $\ell_{3}$ (which is a hyperboloid of one sheet by the general position assumption); ${ }^{2}$ this $3 \times 3$ submatrix corresponds to the quadratic part of the quadric, and thus the discriminant is zero if and only if two eigenvalues are equal, that is, if the hyperboloid is of revolution (since a hyperboloid of one sheet has a canonical equation of the form $\frac{X^{2}}{\delta_{1}^{2}}+\frac{Y^{2}}{\delta_{2}^{2}}-\frac{Z^{2}}{\delta_{3}^{2}}-1=0$ ). This directly proves that the gros facteur is zero if and only if the hyperboloid containing $\ell_{1}, \ell_{2}$, and $\ell_{3}$ is of revolution. Furthermore, this is equivalent to the fact that trisector contains a line; indeed, if the hyperboloid is of revolution, then its axis of revolution is on the trisector, and, conversely, if the trisector contains a line, the gros facteur is zero (since the intersection of the two bisectors is not a nonsingular quartic).

We can now prove the Main Lemma.

\footnotetext{
${ }^{2}$ The equation of the hyperboloid containing $\ell_{1}, \ell_{2}$, and $\ell_{3}$ can easily be computed by solving a linear system obtained by writing that three points on each of the three lines lie on the quadric.
} 
Proof of the Main Lemma Notice that if the hyperboloid containing $\ell_{1}, \ell_{2}$, and $\ell_{3}$ is of revolution, then its center of symmetry, $O$, is equidistant to the three lines. Point $O$ can easily be computed as the intersection of the three planes $P_{1}, P_{2}$, and $P_{3}$, where $P_{1}$ is the bisecting plane of $\ell_{1}$ and the line parallel to $\ell_{1}$ and transversal to $\ell_{2}$ and $\ell_{3}$, and $P_{2}$ and $P_{3}$ are defined similarly (note that $O$ is the center of the parallelepiped shown in Fig. 3 and that $O$ can also be easily computed as the point at which the gradient of the equation of the hyperboloid is zero). The constraint that point $O$ is equidistant to lines $\ell_{1}$ and $\ell_{2}$ then reduces to $(y+a \alpha)(a x+\beta)=0$, which concludes the proof of the Main Lemma.

Note that the above characterization of the gros facteur provides a direct proof of Lemma 5, which essentially states that the gros facteur is nonnegative, because it is the discriminant of a polynomial whose roots are all real (since it is the characteristic polynomial of a real symmetric matrix). Alternatively, this also implies that the gros facteur is a sum of squares [27] and thus nonnegative. ${ }^{3}$

\subsubsection{Computational Proof of the Main Lemma}

We now present our original proof of the Main Lemma, which relies upon modern tools of computer algebra and does not require any specific insight on the geometric meaning of the gros facteur and of the polynomials that appear in the Main Lemma.

\section{Lemma 5 The discriminant $\Delta$ is never negative.}

Proof We prove that the real semi-algebraic set $\mathcal{S}=\left\{\chi=(a, x, y, \alpha, \beta) \in \mathbb{R}^{5} \mid\right.$ $\Delta(\chi)<0\}$ is empty using the RAGLIB Maple package [40], which is based on the algorithm presented in [39]. The algorithm computes at least one point per connected component of such a semi-algebraic set, and we observe that, in our case, this set is empty. Before presenting our computation, we first describe, in the next two paragraphs, the general idea of this algorithm. (This description uses nonelementary notions of algebraic geometry which are not used elsewhere in the paper and can reasonably be skipped.)

Suppose first that $\mathcal{S} \neq \mathbb{R}^{5}$ and let $\mathcal{C}$ denote any connected component of $\mathcal{S}$. We consider here $\Delta$ as a function of all its variables $\chi=(a, x, y, \alpha, \beta) \in \mathbb{R}^{5}$. The algorithm first computes the set of generalized critical values ${ }^{4}$ of $\Delta$ (see [39] for an

\footnotetext{
${ }^{3}$ At the time of submission, we did not succeed to find even an approximation of this sum of squares using SOSTOOLS [36]. However, at the time we wrote the final version of the paper, E. Kaltofen, L. Zhi, and Z. Zhang succeeded to decompose the gros facteur into a sum of squares. They first succeeded to get a numerical approximation of the sum of squares by taking advantage of the structure of the polynomial and reducing the size of the matrices involved in the semidefinite programming (SVD). They were also able to reconstruct the rational coefficients from the approximation using SVD.

${ }^{4}$ Recall that the (real) critical values of $\Delta$ are the values of $\Delta$ at its critical points $\chi$, i.e., the points $\chi$ at which the gradient of $\Delta$ is zero. The asymptotic critical values are similarly defined as, roughly speaking, the values taken by $\Delta$ at critical points at infinity, that is, the values $c \in \mathbb{R}$ such that the hyperplane $z=c$ is tangent to the surface $z=\Delta(\chi)$ at infinity (this definition however only holds for two variables, i.e., $\chi \in \mathbb{R}^{2}$ ). More formally, the asymptotic critical values were introduced by Kurdyka et al. [25] as the
} 
algorithm computing them). The image by $\Delta$ of $\mathcal{C}$ is an interval whose endpoints ${ }^{5}$ are zero and either a negative generalized critical value or $-\infty$. For any $v$ in this interval, there is a point $\chi_{0} \in \mathcal{C}$ such that $\Delta\left(\chi_{0}\right)=v$, and the connected component containing $\chi_{0}$ of the hypersurface $\Delta(\chi)=v$ is included in the connected component $\mathcal{C}$. Hence, a point in $\mathcal{C}$ can be found by computing a point in each connected component of $\Delta(\chi)=v$. It follows that we can compute at least a point in every connected component of the semi-algebraic set $\mathcal{S}$ defined by $\Delta(\chi)<0$ by computing at least one point in every connected component of the real hypersurface defined by $\Delta(\chi)=v$ where $v$ is any value smaller than zero and larger than the largest negative generalized critical value, if any. Now, when $\mathcal{S}=\mathbb{R}^{5}$, that is, $\Delta(p)<0$ for all $p$ in $\mathbb{R}^{5}$, the above computation returns an empty set of points, so we choose a random point $p$ in $\mathbb{R}^{5}$ and return it if $\Delta(p)<0$.

Notice that computing at least one point in every connected component of a hypersurface defined by $\Delta(\chi)=v$ can be done by computing the critical points of the distance function between the surface and a point, say the origin, that is, by solving the system $\Delta(\chi)=v, \chi \times \operatorname{grad}(\Delta)(\chi)=0$. This conceptually simple approach, developed in [38], is, however, not computationally efficient. The efficient algorithm presented in [39] computes instead critical points of projections, combining efficiently the strategies given in [42] and [41].

The computation of at least one point in every connected component of $\mathcal{S}$, using the RAGLIB Maple package, ${ }^{6}$ gives the empty set, implying that $\Delta(\chi) \geq 0$ for all $\chi \in \mathbb{R}^{5}$.

We now prove that the zeros of $\Delta$ are the singular points (that is, the points where all partial derivatives are zero) of the gros facteur.

Lemma 6 The discriminant $\Delta$ is equal to zero if and only if the gros facteur and all its partial derivatives are equal to zero.

Proof As we have seen in the direct proof of the Main Lemma, the discriminant $\Delta$ is equal to zero if and only if the gros facteur is zero. Furthermore, by Lemma 5, the gros facteur is never negative, thus, if there exists a point where the gros facteur vanishes, it is a local minimum of the gros facteur, and thus all its partial derivatives (with respect to $\{a, x, y, \alpha, \beta\})$ are zero.

limits of $\Delta\left(\chi_{k}\right)$ where $\left(\chi_{k}\right)_{k \in \mathbb{N}}$ is a sequence of points that goes to infinity while $\left\|\chi_{k}\right\| \cdot\left\|\operatorname{grad} \chi_{k} \Delta\left(\chi_{k}\right)\right\|$ tends to zero. The generalized critical values are the critical values and asymptotic critical values. The set of generalized critical values contains all the extrema of the function $\mathcal{D}$, even those that are reached at infinity.

${ }^{5}$ Since $\mathcal{S} \neq \mathbb{R}^{5}$, the boundary of $\mathcal{C}$ is not empty and consists of points $\chi$ such that $\mathcal{D}(\chi)=0$. The image of the connected set $\mathcal{C}$ by the continuous function $\mathcal{D}$ is an interval. Hence, zero is an endpoint of the interval $\mathcal{D}(\mathcal{C})$. The other endpoint is either an extremum of $\mathcal{D}$ (and thus a generalized critical value) or $-\infty$.

${ }^{6}$ It should be noted that such computations can be fairly time consuming on a polynomial of the size of $\Delta$ : the computations initially took about 10 hours of elapsed time; they however only took roughly 20 minutes with the version of the package at the time we wrote the final version of the paper. 
Table 1 For the proof of the Main Lemma

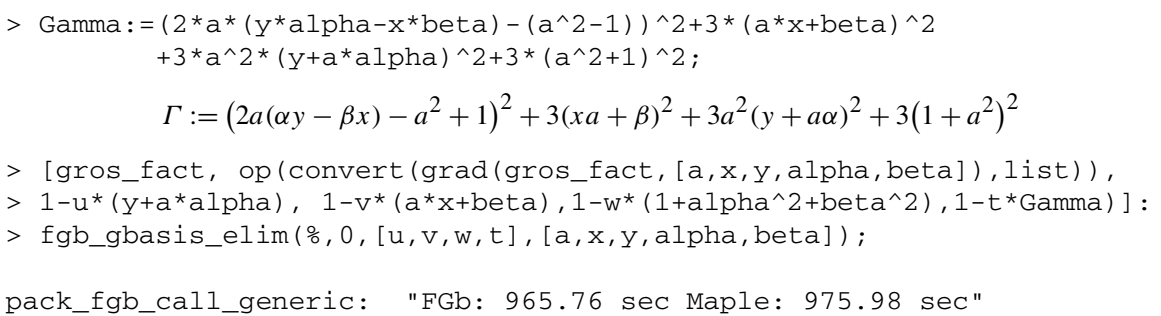

We now present a simple and direct computational proof of the Main Lemma. As we will see, this proof is, however, based on some polynomials whose origins are discussed in Sect. 2.3.

Proof of the Main Lemma By Lemma 6, $\Delta$ is zero if and only if the gros facteur and all its partial derivatives are zero. We prove below that this implies that $(y+a \alpha)(a x+$ $\beta)\left(1+\alpha^{2}+\beta^{2}\right) \Gamma=0$, where

$$
\Gamma=\left(2 a(y \alpha-\beta x)-a^{2}+1\right)^{2}+3(a x+\beta)^{2}+3 a^{2}(y+a \alpha)^{2}+3\left(1+a^{2}\right)^{2} .
$$

As the two terms $\left(1+\alpha^{2}+\beta^{2}\right)$ and $\Gamma$ clearly do not have any real solutions, this proves the lemma. (We discuss later how we found these terms.)

Consider the system in the variables $\{a, x, y, \alpha, \beta, u, v, w, t\}$ that consists of the gros facteur, its partial derivatives, and the four equations

$$
\begin{aligned}
& 1-u(y+a \alpha)=0, \quad 1-v(a x+\beta)=0 \\
& 1-w\left(1+\alpha^{2}+\beta^{2}\right)=0, \quad 1-t \Gamma=0 .
\end{aligned}
$$

The gros facteur and its partial derivatives have a common zero (real or complex) such that $(y+a \alpha)(a x+\beta)\left(1+\alpha^{2}+\beta^{2}\right) \Gamma \neq 0$ if and only if this system has a solution. This follows immediately from the fact that (7) are linear in $u, v, w, t$.

The Gröbner basis of our system is reduced to the polynomial 1 (see Table 1), and thus the system has no solution (over the complex numbers). This concludes the proof.

The real difficulty in this proof of the Main Lemma is, of course, to find (7) that rule out all the components of the set of singular points of the gros facteur. Computing these components is the actual key of the computational proof. We believe that the technique we used can be of some interest to the community as it is rather generic and could be applied to other problems. We thus describe in Sect. 2.3 how these components were computed before finishing the study of the algebraic structure of the trisector in Sect. 2.4. 


\subsection{About the Computational Proof of the Main Lemma}

We show in this section how we computed, for the proof of the Main Lemma, (7) which correspond to hypersurfaces containing the zeros of the discriminant.

We proceed as follows. We start from the system of equations consisting of the gros facteur and all its partial derivatives and use the following techniques to study its set of solutions, or, more precisely, to decompose it into components defined by prime ideals. ${ }^{7}$ This could theoretically be done by a general algorithm computing such a decomposition; however, no currently available software is capable of handling our particular problem, and this is, indeed, a significant research challenge in computer algebra.

If the (reduced) Gröbner basis of some system contains a polynomial which has a factor, say $F$, the solutions of the system splits into two components, one of which such that $F=0$, the other such that $F \neq 0$. We study separately the two components. One is obtained by adding the equation $F$ to the system, and the other is obtained by adding the equation $1-t F$ and eliminating the variable $t$; indeed, there is a oneto-one correspondence between the solutions of the initial system such that $F \neq 0$ and the solutions of the system augmented by $1-t F$. Sometimes, frequently in our case, the component $F \neq 0$ is empty, which corresponds to the situation where the elimination of $t$ results in the polynomial 1 (inducing the equation $1=0$ ). Note that in some cases the system contains a polynomial which is a square, say $F^{2}$; thus the component such that $F \neq 0$ is obviously empty, and we can add $F$ to the system without changing its set of solutions (this however changes the ideal). This operation of adding $F$ to the system frequently adds embedded components to the variety of solutions, which explains why, later on in the process, empty components are frequently encountered when splitting into two components.

Our computations, presented in Table 2 in the Appendix, are performed in Maple [31] using the Gröbner basis package FGb developed by J.-C. Faugère [19]. We use two functions,

$$
\left.f g b \_g \operatorname{basis}(s y s, 0, \operatorname{var} 1, \operatorname{vars} 2) \quad \text { and } \quad f g b \_g \text { basis_elim(sys, } 0, \operatorname{var} 1, \operatorname{var} 2\right),{ }^{8}
$$

that compute Gröbner bases of the system sys; the first uses a degree reverse lexicographic order (DRL) by blocks on the variables of vars 1 and vars 2 (where vars 2 is always the empty set in our computation), and the second one eliminates the variable vars 1 and uses a reverse lexicographic order on the variables of vars 2 . (The second parameter of the functions refers to the characteristic of the field, here 0 .)

We do not show in Table 2 the Gröbner bases, which are too large to be useful, except in the case where the basis is reduced to 1 (when the system has no solution). We instead only report the first operand of each polynomial of the base; the operand $\star$ means that the polynomial is the product of at least two factors; the operand ^ means that the polynomial is a power of some polynomial; and the operand + means that the polynomial is a sum of monomials.

\footnotetext{
${ }^{7}$ An ideal $\mathcal{I}$ is prime if $P Q \in \mathcal{I}$ implies $P \in \mathcal{I}$ or $Q \in \mathcal{I}$.

${ }^{8}$ The function gbasis(sys, DRL(var1, var2), elim) with or without the optional last argument elim can also be used alternatively of these two functions.
} 
Our computation goes as follows. We first simplify our system by considering $a=2$ because otherwise the Gröbner basis computations are too slow and use too much memory to be performed successfully. ${ }^{9}$ We first see after computing $b s_{1}$, the Gröbner basis of our system, that $y+2 \alpha$ appears as a factor of one polynomial. This splits the solutions into those such that $y+2 \alpha=0$ and the others. We will study separately (in Lemma 8) the former set of solutions, and we only consider here the solutions such that $y+2 \alpha \neq 0$. This is done by adding the polynomial $1-u(y+2 \alpha)$ to the system, where $u$ is a new variable; indeed there is a one-to-one correspondence between the solutions of the initial system such that $y+2 \alpha \neq 0$ and the solutions of the resulting system.

The term $y+2 \alpha$ corresponds fairly clearly to the polynomial $y+a \alpha$ with $a=2$, and because of the symmetry of our problem, we also study separately the solutions such that $a x+\beta=0$. Since we assumed $a=2$, we only consider here the solutions such that $2 x+\beta \neq 0$, by adding to the system the polynomial $1-v(2 x+\beta)$. Finally, we also add $1-w\left(1+\alpha^{2}+\beta^{2}\right)$ to the system, without changing its set of real roots; we do this because the term $1+\alpha^{2}+\beta^{2}$ appears in the leading coefficient of $\mathcal{D}(\lambda)$, which suggests that some component of solutions (without any real point) might be included in $1+\alpha^{2}+\beta^{2}$ (it should be noted that adding this polynomial to the system changes the resulting Gröbner basis, which shows that this indeed removes some imaginary component from the system). We compute the Gröbner basis, $b s_{2}$, of that system, eliminating the variables $u, v, w$, which gives a system of four polynomials of degree six.

We then compute the Gröbner basis of $b s_{2}$, eliminating the variable $x$. This gives a basis $b s_{3}$ which is reduced to one polynomial of the form $P^{2}$. We thus add $P$ to the system $b s_{2}$ (we do not add it to $b s_{3}$ since $b s_{3}$ does not depend on $x$ ). The Gröbner basis, $b s_{4}$, of the new system contains several polynomials that are products of factors. We see that if we add to the system the constraint that the third factor of the first polynomial is not zero, the resulting system has no solution. We thus add this factor to the system and compute its Gröbner basis $b s_{5}$. We operate similarly to get $b s_{6}$. The basis $b s_{6}$ contains no product or power, and we compute its Gröbner basis, $b s_{7}$, eliminating $y$ (eliminating $x$ gives no interesting basis). The last polynomial of $b s_{7}$ is a power, and we proceed as before to get $b s_{8}$. We proceed similarly until we get to the basis $b s_{12}$. (Note that the factor $y+2 \alpha$ reappears in $b s_{10}$ and is removed similarly as in the beginning of the process.)

The basis $b s_{12}$ consists of three polynomials of degree four (which is a simplification over $b s_{2}$ consisting of four polynomials of degree six). We observe that the last polynomial of $b s_{12}$ is

$$
\Gamma_{2}=(4 y \alpha-4 \beta x-3)^{2}+3(2 x+\beta)^{2}+12(y+2 \alpha)^{2}+75,
$$

which is always positive over the reals.

\footnotetext{
${ }^{9}$ The choice of specializing variable $a$ follows from the observation that all the polynomials are almost homogeneous in the other variables and from our experience that Gröbner computations are usually much easier with homogeneous or almost homogeneous systems.
} 
We have thus proved that all the complex solutions of the initial system such that $a=2$ (the gros facteur and all its partial derivatives) satisfy $\left(1+\alpha^{2}+\beta^{2}\right)(y+$ $2 \alpha)(2 x+\beta) \Gamma_{2}=0$.

Finally, to get the polynomial $\Gamma$ of formula (6), we performed the same computation with $a=3$ and $a=5$ and guessed $\Gamma$ as an interpolation of the polynomials $\Gamma_{2}$, $\Gamma_{3}$, and $\Gamma_{5}$.

Note that all the computation for a fixed $a$ takes roughly eight minutes of elapsed time.

Remark 7 All the computations from $b s_{2}$ to $b s_{12}$ amounts to finding polynomials that have a power which is a combination of the elements of $b s_{2}$ (i.e., which are in the radical of the ideal generated by $b_{2}{ }^{10}$ ). Thus these computations would be advantageously replaced by a program computing the radical of an ideal. Unfortunately, all available such programs fail on the ideal generated by $b s_{2}$ either by exhausting the memory or by running unsuccessfully during several days and ending on an error. It is therefore a challenge to improve these programs in order to do this computation automatically.

\subsection{Structure of the Trisector: Conclusion}

We proved in the Main Lemma that the discriminant $\Delta$ is equal to zero only if $y+a \alpha=0$ or $a x+\beta=0$. We prove in this section that if $\Delta=0$, the trisector is a nonsingular cubic and a line that do not intersect. We then show that the trisector always contains four simple real points at infinity and conclude that the trisector is always homeomorphic to four lines that do not pairwise intersect.

Lemma 8 The discriminant $\Delta$ is equal to zero if and only if

$$
\begin{aligned}
& y=-a \alpha \quad \text { and } \quad x=\frac{\beta\left(2 a^{2}+1\right) \pm 2 \sqrt{a^{2}\left(1+a^{2}\right)\left(\alpha^{2}+\beta^{2}+1\right)}}{a}, \quad \text { or } \\
& x=-\frac{\beta}{a} \quad \text { and } \quad y=\frac{\alpha\left(2+a^{2}\right) \pm 2 \sqrt{\left(1+a^{2}\right)\left(\alpha^{2}+\beta^{2}+1\right)}}{a} .
\end{aligned}
$$

Proof We refer to Table 3 in the Appendix for the computations. By the Main Lemma, $\Delta=0$ implies $y+a \alpha=0$ or $a x+\beta=0$. Substituting $y$ by $-a \alpha$ in $\Delta$ gives an expression of the form $f_{0} f_{1}^{2}$. Similarly, substituting $x$ by $-\beta / a$ in $\Delta$ gives an expression of the form $g_{0} g_{1}^{2}$ (recall that $a \neq 0$ since the lines are not coplanar, by assumption). It follows that $\Delta=0$ if and only if $y+a \alpha=f_{i}=0$ or $a x+\beta=g_{i}=0$ for $i=0$ or 1 .

The $f_{i}$ and $g_{i}$ are polynomials of degree two in $x$ and $y$, respectively. Solving $f_{1}=0$ in terms of $x$ directly yields that the system

$$
y+a \alpha=f_{1}=0
$$

${ }^{10}$ The radical of an ideal $\mathcal{I}$ is the ideal $\left\{P \mid P^{n} \in \mathcal{I}\right.$ for some $\left.n \in \mathbb{N}\right\}$. 
is equivalent to (8). Similarly, solving $g_{1}=0$ in terms of $y$ yields that the system

$$
a x+\beta=g_{1}=0
$$

is equivalent to (9).

We now show that the solutions of $y+a \alpha=f_{0}=0$ are included in the set of solutions of (9). The polynomial $f_{0}$ is the sum of two squares. It follows that $y+a \alpha=$ $f_{0}=0$ if and only if

$$
y+a \alpha=a^{2} \alpha^{2}-1+a \beta x=a x+\beta=0 .
$$

We show below that the polynomials of (11) are included in the ideal generated by the polynomials of (12). This implies that (11) is, roughly speaking, less constrained than (12) and that the set of solutions of (11) contains the solutions of (12). Hence the solutions of $y+a \alpha=f_{0}=0$ are contained in the set of solutions of (11) and thus in the set of solutions of (9).

We prove that the polynomials of (11) are included in the ideal generated by the polynomials of (12) by showing that the normal form of every polynomial of (11) with respect to the Gröbner basis of the polynomials of (12) is zero. This is done using the function normalf (of Maple), which computes the normal form of a polynomial with respect to a Gröbner basis.

We prove similarly that the solutions of $a x+\beta=g_{0}=0$ are included in the set of solutions of (10) and thus of (8), which concludes the proof.

Remark 9 Note that by symmetry with respect to the $X Y$-plane and by changing the sign of $a, \alpha$, and $\beta$, the set of three input lines $\ell_{1}, \ell_{2}, \ell_{3}$ is invariant, the two components of (8) exchange (i.e., the components corresponding to $+2 \sqrt{\cdots}$ and $-2 \sqrt{\cdots}$ exchange), and the two components of (9) exchange.

Similarly, by exchanging the $X$ and $Y$-coordinates, $x$ and $y, \alpha$ and $\beta$, and changing $a$ into $1 / a$, the set of three input lines is also invariant, and each component of (8) is changed to a component of (9), and conversely.

Lemma 10 If $\Delta=0$, the trisector of $\ell_{1}, \ell_{2}$, and $\ell_{3}$ consists of a nonsingular cubic and a line that do not intersect in real space.

Proof By Lemma 8, $\Delta=0$ if and only if system (8) or (9) is satisfied. By symmetry of the problem (see Remark 9), we only need to consider one of the components of (8) and (9). Hence, it is sufficient to show that

$$
y=-a \alpha, \quad x=\frac{\beta\left(2 a^{2}+1\right)}{a}+2 \sqrt{\left(1+a^{2}\right)\left(\alpha^{2}+\beta^{2}+1\right)}
$$

implies that the trisector consists of a nonsingular cubic and a line that do not intersect. We assume in the following that $\Delta=0$ and that system (13) is satisfied. We refer to Table 4 for the computations.

We first show that the characteristic polynomial of the pencil generated by the bisectors is always strictly positive. Note first that the characteristic polynomial is not always negative (see [30]). It is thus sufficient to prove that it is never zero, 
or equivalently, that its product with its algebraic conjugate (obtained by changing the sign of $\sqrt{\left(1+a^{2}\right)\left(\alpha^{2}+\beta^{2}+1\right)}$ ) is never zero. This product is a polynomial $T$ in $a, \alpha, \beta, \lambda$ which can easily be factored in the square of a degree-two polynomial in $\lambda$; furthermore, this degree two polynomial has no real root because its discriminant is the product of a negative term $\left(-\left(1+a^{2}\right)^{2}\left(1+\alpha^{2}+\beta^{2}\right)\right)$ and a term whose sum and product with its algebraic conjugate (obtained, as above, by changing the sign of the square root) is a strictly positive sum of squares. Note that we can also prove that $T$ is always strictly positive by computing, similarly as in the proof of Lemma 5, at least one point per connected component of the real semi-algebraic set $\left\{\chi=(a, \alpha, \beta, \lambda) \in \mathbb{R}^{4} \mid T(\chi)-\frac{1}{2}<0\right\}$; the resulting set of points is empty, hence $T(\chi)$ is always greater or equal to $1 / 2$. It thus follows that the characteristic polynomial of the pencil is always strictly positive.

Since the characteristic polynomial $\mathcal{D}(\lambda)$ is always strictly positive and its discriminant $\Delta$ is zero, $\mathcal{D}(\lambda)$ admits two (conjugate) double imaginary roots. Let $\lambda_{1}$ and $\lambda_{2}$ denote these two roots. Recall that $\mathcal{D}(\lambda)=\operatorname{det} P(\lambda)$ with $P(\lambda)=\lambda Q_{1,2}+Q_{1,3}$ where $Q_{i, j}$ is the matrix associated with the hyperbolic paraboloid $\mathcal{H}_{i, j}$. It follows from the classification of the intersection of quadrics [14, Table 1] that either (i) $P\left(\lambda_{1}\right)$ and $P\left(\lambda_{2}\right)$ are of rank 3 , and the trisector $\mathcal{H}_{1,2} \cap \mathcal{H}_{1,3}$ consists of a cubic and a line that do not intersect, or (ii) $P\left(\lambda_{1}\right)$ and $P\left(\lambda_{2}\right)$ are of rank 2 , and the trisector consists of two secant lines.

We now prove that $P\left(\lambda_{1}\right)$ and $P\left(\lambda_{2}\right)$ are of rank 3 . We compute the Gröbner basis of all the $3 \times 3$ minors of $P(\lambda)$ and of the polynomial $1-t \Psi$ with

$$
\Psi=\left(1+a^{2}\right)\left(1+\alpha^{2}+\beta^{2}\right)(a x-y-\beta+a \alpha)(y+a x-a \alpha-\beta) .
$$

The basis is equal to 1 , thus the $3 \times 3$ minors of $P(\lambda)$ are not all simultaneously equal to zero when $\Psi \neq 0$. Furthermore, $\Psi \neq 0$ for any $x, y, a, \alpha, \beta$ in $\mathbb{R}$ such that the lines $\ell_{1}, \ell_{2}$, and $\ell_{3}$ are pairwise skew (see (4) and the proof of Lemma 6). Thus the rank of $P(\lambda)$ is at least 3 . The rank of $P\left(\lambda_{i}\right), i=1,2$, is thus equal to 3 since $\operatorname{det} P\left(\lambda_{i}\right)=0$. We can thus conclude that when $\Delta=0$, the trisector consists of a cubic and a line that do not intersect in real space.

It remains to argue that the cubic is nonsingular. First, note that the cubic cannot be planar since, in that case, it would be a conic (the planar section of a quadric). The result follows from the well-known fact that every skew cubic is nonsingular, which we prove again for completeness. Consider a singular cubic and a plane defined by (one of) its singular point(s) and any two other of its points. The cubic intersects this plane in at least these three points, that is, in at least four points counted with multiplicity, which implies that the cubic is contained in the plane.

We now state a proposition that shows that the trisector admits four asymptotes that are pairwise skew and gives a geometric characterization of their directions.

Proposition 11 The trisector of $\ell_{1}, \ell_{2}$, and $\ell_{3}$ intersects the plane at infinity in four real simple points. Furthermore, the four corresponding asymptotes are parallel to the four trisector lines of three concurrent lines that are parallel to $\ell_{1}, \ell_{2}$, and $\ell_{3}$, respectively. 
Proof The trisector is the intersection of two hyperbolic paraboloids. Any hyperbolic paraboloid contains two lines at infinity. Hence the intersection, at infinity, of any two distinct hyperbolic paraboloids is the intersection of two pairs of lines. The intersection of these two pairs of lines consists of exactly four simple real points unless the point of intersection of the two lines in one pair lies on one line of the other pair. We show that this cannot happen under our assumptions.

The intersection with the plane at infinity of the bisector of lines $\ell_{1}$ and $\ell_{2}$ consists of the lines at infinity in the pair of planes of equation $X Y=0$ (the homogeneous part of highest degree in (2)). This pair of plane is the bisector of the two concurrent lines that are parallel to $\ell_{1}$ and $\ell_{2}$, respectively. Note that the lines at infinity in this pair of planes are invariant by translation of the planes. We thus get that the lines at infinity of the bisector of any two lines $\ell_{i}$ and $\ell_{j}$ are the lines at infinity in the pair of planes that is the bisector to any two concurrent lines that are parallel to $\ell_{i}$ and $\ell_{j}$, respectively.

It follows that the points at infinity on the trisector of $\ell_{1}, \ell_{2}$, and $\ell_{3}$ are the points at infinity on the trisector lines (the intersection of bisector planes) of three concurrent lines that are parallel to $\ell_{1}, \ell_{2}$, and $\ell_{3}$, respectively. It remains to show that this trisector consists of four distinct lines.

Let $\ell_{1}^{\prime}, \ell_{2}^{\prime}$, and $\ell_{3}^{\prime}$ be the three concurrent lines through the origin that are parallel to $\ell_{1}, \ell_{2}$, and $\ell_{3}$, respectively, and suppose, for a contradiction, that their trisector does not consist of four distinct lines. This implies that the line of intersection of the two bisector planes of two lines, say $\ell_{1}^{\prime}$ and $\ell_{2}^{\prime}$, is contained in one of the bisector planes of two other lines, say $\ell_{1}^{\prime}$ and $\ell_{3}^{\prime}$. The intersection of the bisector planes of $\ell_{1}^{\prime}$ and $\ell_{2}^{\prime}$ is the $Z$-axis. It follows that one of the bisector planes of $\ell_{1}^{\prime}$ and $\ell_{3}^{\prime}$ is vertical, hence $\ell_{1}^{\prime}$ and $\ell_{3}^{\prime}$ are symmetric with respect to a vertical plane, and thus $\ell_{3}^{\prime}$ is contained in the $X Y$-plane. Therefore, $\ell_{1}^{\prime}, \ell_{2}^{\prime}$, and $\ell_{3}^{\prime}$ lie in the $X Y$-plane, contradicting the general position assumption, which concludes the proof.

Theorem 12 The trisector of three lines in general position consists of four infinite smooth branches of a nonsingular quartic or of a nonsingular cubic and a line that do not intersect in real projective space.

Proof As mentioned at the beginning of Sect. 2.2, the trisector of three lines consists of a smooth quartic unless the discriminant $\Delta$ is zero. Lemma 10 and Proposition 11 thus yield the result.

\section{Properties of the Voronoi Diagram}

We present here some fundamental properties of the Voronoi diagram. We will show how the four branches of the trisector of three lines can be labeled and then present two fundamental properties of the trisector.

\subsection{Preliminaries}

We start with the following important proposition. Recall first that the set of triplets of pairwise skew lines admits two connected components [49]. We prove here that this result also holds for the set of labeled triplets of lines in general position (that is, pairwise skew and not all parallel to a common plane). 


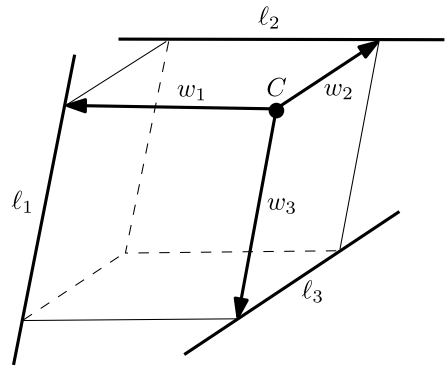

(a)

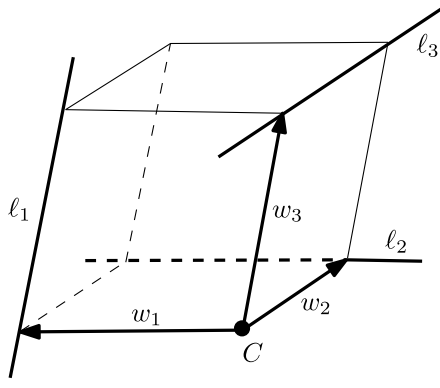

(b)

Fig. 3 Two instances of parallelepipeds formed by $\ell_{1}, \ell_{2}$, and $\ell_{3}$ and of the associated frames $\left(C, w_{1}, w_{2}, w_{3}\right)$

Proposition 13 The set of labeled triplets of lines in general position admits two connected components. Moreover, the triplets in one component are mirror images of the triplets in the other component.

Proof We prove this proposition by proving that there is a one-to-one correspondence between the set of labeled triplets of lines in general position and the set of affine frames, which admits two components, the set of frames with positive orientations and those with negative orientations.

Consider three lines $\ell_{1}, \ell_{2}$, and $\ell_{3}$ in general position and refer to Fig. 3. For the three choices of pairs of lines $\ell_{i}, \ell_{j}$, consider the plane containing $\ell_{i}$ and parallel to $\ell_{j}$, the plane containing $\ell_{j}$ and parallel to $\ell_{i}$, and the region bounded by these two parallel planes. The general position assumption implies that these regions have nonempty interiors and that no three planes are parallel. The intersection of these three regions thus defines a parallelepiped. By construction, each of the lines $\ell_{1}, \ell_{2}$, and $\ell_{3}$ contains an edge of that parallelepiped. These lines are pairwise skew; thus exactly two vertices of the parallelepiped are not on the lines. Each of these two points induces an affine frame centered at the point and with basis $\left(w_{1}, w_{2}, w_{3}\right)$, the three edges of the parallelepiped oriented from the point to the lines $\ell_{1}, \ell_{2}$, and $\ell_{3}$, in this order. We consider the frame such that $w_{i}$ is parallel to $\ell_{i+1}$ (modulo 3 ). Conversely, a frame $\left(C, w_{1}, w_{2}, w_{3}\right)$ defines uniquely a parallelepiped and three lines $\ell_{1}, \ell_{2}$, and $\ell_{3}$ such that $\ell_{i}$ goes through the vertex $C+w_{i}$ and is parallel to $w_{i-1}$ (modulo 3).

We consider in the following any three lines $\ell_{1}, \ell_{2}$, and $\ell_{3}$ in general position (pairwise skew and not all parallel to a common plane) and an associated Cartesian coordinate system $(X, Y, Z)$ such that the $Z$-axis is the common perpendicular of $\ell_{1}$ and $\ell_{2}$, the origin is the point on the $Z$-axis equidistant to $\ell_{1}$ and $\ell_{2}$, and such that the $X$-and $Y$-axes are the two bisector lines, in the plane through the origin and perpendicular to the $Z$-axis, of the projection of $\ell_{1}$ and $\ell_{2}$ onto this plane. Note that the orientations of the axes are not specified (except for the fact that the frame has a positive orientation) and that the $X$ - and $Y$-axes can be exchanged. 


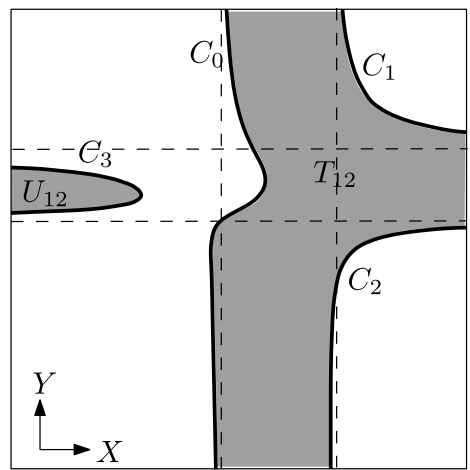

(a)

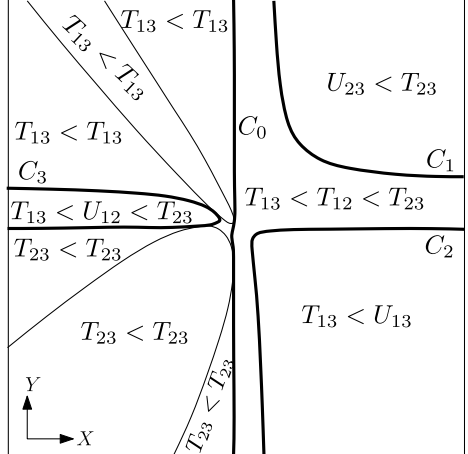

(b)

Fig. 4 (a) The shaded region is the projection of the two-dimensional Voronoi cell $V_{12}$ onto the $X Y$-plane. (b) Vertical ordering of the sheets of the connected components of the two-dimensional Voronoi diagram cells above each region induced by the projection of the trisector and the silhouette curves of the bisectors; the ordering over the small cell in the middle is $T_{13}<T_{13}<T_{23}<T_{23}$ (i.e., a vertical line over that cell intersects twice $T_{13}$ and twice $T_{23}$ in that order)

\subsection{Labeling of the Four Branches of the Trisector}

We prove here the following proposition which states two properties, one on the asymptotes of the trisector and one on the incidence relations between cells, which, together, yield an unambiguous labeling of the components of the trisector.

Let $V_{i j}$ denote the two-dimensional Voronoi cell of lines $\ell_{i}$ and $\ell_{j}$, and let $U_{i j}$ and $T_{i j}$ denote the connected components of $V_{i j}$ that are bounded by one and three arcs of the trisector, respectively (see Fig. 4(a)).

Proposition 14 Exactly one of the four branches of the trisector of three lines in general position admits only one asymptote. Let $C_{0}$ denote this branch. Each cell $U_{i j}$ is bounded by a branch distinct from $C_{0}$ and every such branch bounds a cell $U_{i j}$. Let $C_{k}, k=1,2,3$, denote the branches of the trisector that bound the component $U_{i j}, i, j \neq k$. The labeling of the four branches of the trisector by $C_{0}, \ldots, C_{4}$ is unambiguous.

Note that differentiating between $C_{1}$ and $C_{2}$ cannot be done, as far as we know, by only looking at the cell $V_{12}$ (see Fig. 4(a)) but can be done by looking at the other cells $V_{13}$ and $V_{23}$. More precisely, differentiating between $C_{1}$ and $C_{2}$ in Fig. 4(a) can be done by computing (as described in the proof of Lemma 16) a vertical ordering of the sheets of the components $U_{i j}$ and $T_{i j}$; the branch $C_{k}$ is then characterized as the branch for which $U_{i j}$ appears only on one of its sides (see Fig. 4(b)).

We prove two lemmas that, together, prove Proposition 14.

Lemma 15 Exactly one of the four branches of the trisector of three lines in general position admits only one asymptote.

Proof By Proposition 11, the trisector admits four distinct asymptotes for all triplets of lines in general position. It follows that the property that exactly one of the 
branches of the trisector has only one asymptote is invariant by continuous deformation on the set of triplets of lines in general position. Moreover, if this property is verified for the trisector of a triplet of lines, it is also verified for a mirror image of the trisector and thus for the trisector of a mirror image of the three lines. The result thus follows from Proposition 13 and from the observation that the property is verified on one particular example.

This property can be observed in Fig. 4(a) and it can easily be proved as follows. Consider any three lines, in general position, whose trisector consists in a cubic and a line (three such lines exist by Lemmas 8 and 10). The line is one branch of the trisector that admits only one asymptote. On the other hand, since the cubic consists of only one connected component in projective space and it intersects the plane at infinity in three real simple points (by Proposition 11), each of its three branches has two asymptotes.

We denote by $C_{0}$ the branch of the trisector that admits only one asymptote (see Fig. 4(a)).

Lemma 16 Each cell $U_{i j}$ is bounded by a branch of the trisector distinct from $C_{0}$ and every such branch bounds a cell $U_{i j}$.

Proof This property is invariant by continuous deformation on the set of triplets of lines in general position. Moreover, similarly as in the previous proof, if this property is verified for the Voronoi diagram of a triplet of lines, it is also verified for a mirror image of the Voronoi and thus for the Voronoi diagram of a mirror image of the three lines. By Proposition 13, it is thus sufficient to prove it for any three given lines in general position, $\ell_{1}, \ell_{2}, \ell_{3}$, as defined in Sect. 2.1. We consider in the $X Y$-plane the arrangement of the (orthogonal) projection of the trisector and of the silhouette curves (viewed from infinity in the $Z$-direction) of the bisectors (see Fig. 4(b)); these silhouette curves consist of only two parabolas since the bisector of lines $\ell_{1}$ and $\ell_{2}$ has no such silhouette (its equation has the form $Z=\gamma X Y$-see (2)—and thus any vertical line intersects it). By construction, for all vertical lines intersecting one given (open) cell of this arrangement, the number and ordering of the intersection points between the vertical line and all the pieces of the three bisectors that are bounded by the trisector is invariant. For any point of intersection, we can easily determine (by computing distances) whether the point lies on a Voronoi cell $V_{i j}$. We can further determine whether the point belongs to the component $U_{i j}$ or $T_{i j}$ by using the linear separation test described in Section 6. We thus report the ordering of the sheets of the components $U_{i j}$ and $T_{i j}$ above each cell of the arrangement in the $X Y$-plane for a given example; see Fig. 4(b).

We can now observe that there is a one-to-one correspondence between the three branches of the trisector distinct from $C_{0}$ and the components $U_{12}, U_{13}$, and $U_{23}$ such that the component appears only on one side of the corresponding branch. ${ }^{11}$ It follows that each of the branches distinct from $C_{0}$ bounds a cell $U_{i j}$.

\footnotetext{
${ }^{11}$ Namely, $U_{13}$ (resp. $U_{23}$ and $U_{12}$ ) appears on only one side of the lower-right (resp. upper-right and left-most) branch.
} 
Proof of Proposition 14 Lemmas 15 and 16 state the first two properties of Proposition 14 . Furthermore, since $U_{i j}$ is, by definition, bounded by only one arc of the trisector, Lemmas 15 and 16 directly yield that the labeling of the four branches of the trisector by $C_{0}, \ldots, C_{4}$ is unambiguous.

\subsection{Properties of the Trisector}

We now prove two important properties of trisector of the Voronoi diagram of three lines in general position. In particular, we prove the Monotonicity Property in Proposition 18.

Proposition 17 The orthogonal projection of the trisector of $\ell_{1}, \ell_{2}$, and $\ell_{3}$ onto the $X Y$-plane has two asymptotes parallel to the $X$-axis and two asymptotes parallel to the $Y$-axis.

Proof By Proposition 11, the four asymptotes of the trisector are parallel to the four trisector lines of three concurrent lines parallel to $\ell_{1}, \ell_{2}$, and $\ell_{3}$. The bisector to two lines through the origin and parallel to $\ell_{1}$ and $\ell_{2}$ is the pair of planes of equation $X Y=0$. Hence the asymptotes of the trisector are parallel to lines that lie in the pair of planes $X Y=0$. The orthogonal projection of the asymptotes on the $X Y$-plane are thus parallel to the $X$ or $Y$-axis. It follows that the number of asymptotes (in projection) that are parallel to the $X$-axis (resp. $Y$-axis) is invariant by continuous deformation on any connected set of triplets of lines in general position. Moreover, as in the proofs of the two previous lemmas, if this property is verified for the trisector of a triplet of lines, it is also verified for the trisector of a mirror image of the three lines. The result thus follows from Proposition 13 and from the fact that, on a particular example (see Fig. 4(a)), there are two asymptotes parallel to the $X$-axis and two others parallel to the $Y$-axis.

We assume in the following that the asymptote of $C_{0}$ is parallel to the $Y Z$-plane (as in Fig. 4(a)) by exchanging, if necessary, the roles of $X$ and $Y$.

Proposition 18 Every branch of the trisector of $\ell_{1}, \ell_{2}$, and $\ell_{3}$ is strictly monotonic with respect to the $Y$-direction (or every branch is strictly monotonic with respect to the $X$-direction).

Proof Let $\mathcal{P}$ denote any plane parallel to the $X Z$-plane. The $\operatorname{arc} C_{0}$ intersects plane $\mathcal{P}$ an odd number of times (counted with multiplicity) since $C_{0}$ has only one asymptote (Proposition 14) which is parallel to the $Y Z$-plane. Furthermore, by Proposition 17, the trisector has two other asymptotes parallel to the $X Z$-plane. Hence plane $\mathcal{P}$ intersects the trisector in two points at infinity and $C_{0}$ an odd number of times (in affine space). The trisector thus intersects $\mathcal{P}$ in at least three points in real projective space. There are thus four intersection points (in real projective space) since there are four intersection points in complex space (since the trisector is of degree four), and if there was an imaginary point of intersection, its conjugate would also be an intersection point (since the equations of the plane and quadrics have real coefficients) giving five points of intersection. 
Therefore the trisector intersects plane $\mathcal{P}$ in two points in $\mathbb{R}^{3}$ (counted with multiplicity), one of which lies on $C_{0}$. Since there are an odd number of intersection points on $C_{0}$, plane $\mathcal{P}$ intersects $C_{0}$ exactly once, and any other branch exactly once (counted with multiplicity).

\section{Topology of the Voronoi Diagram}

We prove in Theorem 19 that the topology of the Voronoi diagram of three lines in general position is invariant. Theorem 1 will thus follow from Theorem 12 and from the computation of an example of a two-dimensional cell of the Voronoi diagram (for instance, the one shown in Fig. 1). Let $\mathcal{S}$ denote the set of triplets of lines in general position.

Theorem 19 The topology of the Voronoi diagram of three lines in general position is constant. More precisely, given two triplets of lines in one connected component of $\mathcal{S}$, there is a continuous path in $\mathcal{S}$ between them which induces a continuous deformation of every cell of the Voronoi diagram, preserving the topology of the cells and the incidence relations between them. Moreover, the Voronoi diagram of three lines in one connected component of $\mathcal{S}$ is a mirror image of the Voronoi diagram of three lines in the other connected component of $\mathcal{S}$.

Proof The general idea of the proof is as follows. Consider three lines in general position and a bisector of two of them. The bisector is a hyperbolic paraboloid which is homeomorphic to a plane. The trisector lies on the bisector, and it is homeomorphic to four lines that do not pairwise intersect, by Theorem 12. Hence the topology of the regions that lie on the bisector and are bounded by the trisector is invariant by continuous deformation on any connected component of $\mathcal{S}$. It follows that the topology of the two-dimensional cells of the Voronoi diagram is invariant by such a continuous deformation. The Voronoi diagram is defined by the embedding in $\mathbb{R}^{3}$ of its twodimensional cells, hence its topology is also invariant by continuous deformation on any connected component of $\mathcal{S}$. Finally, the topology is also invariant over $\mathcal{S}$ since, by Proposition 13, the Voronoi diagram of three lines in one connected component of $\mathcal{S}$ is a mirror image of the Voronoi diagram of three lines in the other connected component of $\mathcal{S}$.

To be more precise, we now show that any continuous path in $\mathcal{S}$ between any two triplets of lines in one connected component of $\mathcal{S}$ induces a continuous deformation of every cell of the Voronoi diagram, preserving the topology of the cells and the incidence relations between them.

Consider two triplets of lines, $\left(\ell_{1}, \ell_{2}, \ell_{3}\right)$ and $\left(\ell_{1}^{\prime}, \ell_{2}^{\prime}, \ell_{3}^{\prime}\right)$, in one connected component of $\mathcal{S}$ and a homotopy between them, i.e., a continuous application $\varphi: t \mapsto$ $\varphi(t)=\left(\ell_{1}(t), \ell_{2}(t), \ell_{3}(t)\right)$ of the interval $[0,1]$ into $\mathcal{S}$ such that $\varphi(0)=\left(\ell_{1}, \ell_{2}, \ell_{3}\right)$ and $\varphi(1)=\left(\ell_{1}^{\prime}, \ell_{2}^{\prime}, \ell_{3}^{\prime}\right)$. Without loss of generality, we may choose for $\left(\ell_{1}, \ell_{2}, \ell_{3}\right)$ the triplet of Fig. 1.

Consider now an orthonormal frame $\mathcal{F}(t)$ such that the $Z$-axis is the common perpendicular to $\ell_{1}(t)$ and $\ell_{2}(t)$, the origin of the frame is the point of the $Z$-axis equidistant to $\ell_{1}(t)$ and $\ell_{2}(t)$, and the $X$ - and $Y$-axes are the bisectors of the projections of 
$\ell_{1}(t)$ and $\ell_{2}(t)$ onto the plane orthogonal to the $Z$-axis. Note that this coordinate system is, up to a possible change of orientation of the axes and a possible exchange of the $X$ - and $Y$-axes, the one we considered in Sects. 2 and 3 and which has been used to draw Fig. 1. When the parameter $t$ of the homotopy varies from 0 to 1 , the lines vary continuously, and thus the frame $\mathcal{F}(t)$ can be defined to vary continuously in terms of $t$.

By Lemma 15 and Propositions 17 and 18, for any $t$ in $[0,1]$, each of the branches of the trisector is monotonic with respect to either the $X$ or the $Y$-direction, but not both. Furthermore, the set of $t$ for which each branch is monotonic with respect to the $X$-direction (resp. the $Y$-direction) is closed (since the lines and $\mathcal{F}(t)$ vary continuously in terms of $t$ ). Hence, each branch of the trisector is monotonic in $X$ for all $t$ or is monotonic in $Y$ for all $t$. Therefore, by exchanging, if needed, $X$ and $Y$ in all frames $\mathcal{F}(t)$, we may suppose that each of the four branches of the trisector of $\ell_{1}(t)$, $\ell_{2}(t)$, and $\ell_{3}(t)$ is monotonic with respect to the $Y$-direction.

In the coordinate system $\mathcal{F}(t)$, the bisector of $\ell_{1}(t)$ and $\ell_{2}(t)$ has the equation $Z=\alpha(t) X Y$ (see the proof of Lemma 4). Substituting $Z$ by $\alpha(t) X Y$ in the equation of the bisector of $\ell_{2}(t)$ and $\ell_{3}(t)$ in the coordinate system $\mathcal{F}(t)$, we get an equation of degree 2 in each of the variables $X$ and $Y$. Solving it in $X$, we get a parameterization of the form $X=\Upsilon^{ \pm}(Y, t)$ with $\Upsilon^{ \pm}(Y, t)=\frac{-P_{1}(Y, t) \pm \sqrt{P_{1}(Y, t)^{2}-4 P_{0}(Y, t) P_{2}(Y, t)}}{2 P_{2}(Y, t)}$, where $P_{0}, P_{1}$, and $P_{2}$ are polynomials of degree 2 in $Y$, which depend continuously on $t$ (since the frame $\mathcal{F}(t)$ and the equations, in any fixed frame, of the bisectors depend continuously on $t$ ).

Notice first that $P_{4}(Y, t)=P_{1}(Y, t)^{2}-4 P_{0}(Y, t) P_{2}(Y, t)$ is always positive. Indeed, it is always nonnegative since one of the branches of the trisector of $\ell_{1}(t)$, $\ell_{2}(t)$, and $\ell_{3}(t)$ is defined for all $Y$ in $\mathcal{F}(t)$ (since each branch is monotonic in $Y$ and one of them has only one asymptote, by Lemma 15). It thus follows from the fact that the trisector has no real singular point (Theorem 12) that $P_{4}(Y, t)$ is always positive. Notice also that, for any $t$ in $[0,1], P_{2}(Y, t)$ has two distinct real roots by Proposition 17.

Since $P_{4}(Y, t)$ is always positive, the branch $C_{0}(t)$ of $\ell_{1}(t), \ell_{2}(t)$, and $\ell_{3}(t)$ (see Proposition 14) is parameterized by $X=\Upsilon^{-}(Y, t)$ or by $X=\Upsilon^{+}(Y, t)$ (but not by a combination of both). Thus, by changing, if needed, the signs of $P_{0}, P_{1}$, and $P_{2}$, we may suppose that $C_{0}(0)$ is parameterized by $X=\Upsilon^{-}(Y, 0)$. This implies, by continuity, that the branch $C_{0}(t)$ is parameterized, in the frame $\mathcal{F}(t)$, by $X=\Upsilon^{-}(Y, t)$, while the other branches are parameterized by $X=\Upsilon^{+}(Y, t)$ and the position of $Y$ with respect to the two roots of $P_{2}(Y, t)$.

The study of the Voronoi diagram of $\ell_{1}(0), \ell_{2}(0)$, and $\ell_{3}(0)$ (see Figs. 1 and 4(a)) thus implies that the region, denoted $\mathcal{R}_{12}(t)$, of the Voronoi diagram consisting in the points which are at the same distance of the lines $\ell_{1}(t)$ and $\ell_{2}(t)$ and closer than to $\ell_{3}(t)$ consists, when $t=0$, in two open semi-algebraic sets defined in $\mathcal{F}(0)$ by (i) $Z=\alpha(0) X Y, X<\Upsilon^{+}(Y, 0)$, and $Y$ between the two roots of $P_{2}$, and by (ii) $Z=$ $\alpha(0) X Y, X>\Upsilon^{-}(Y, 0)$, and, when $Y$ is outside the two roots of $P_{2}, X<\Upsilon^{+}(Y, 0)$.

Now, since the objects we are considering depend continuously on $t$, including the distance from a point to one of the lines (note that the distance function is defined independently of $\mathcal{F}(t)$ ), the Voronoi region $\mathcal{R}_{12}(t)$ is defined, similarly, by the two open semi-algebraic sets defined in $\mathcal{F}(t)$ by (i) $Z=\alpha(t) X Y, X<\Upsilon^{+}(Y, t)$, and $Y$ 
between the two roots of $P_{2}$, and by (ii) $Z=\alpha(t) X Y, X>\Upsilon^{-}(Y, t)$, and, when $Y$ is outside the two roots of $P_{2}, X<\Upsilon^{+}(Y, t)$.

Note that, in the case where the trisector is decomposed, for some value of $t$, into a cubic and a line, nothing changes in what precedes, the only difference being that the square root is a polynomial and that the parameterization of $C_{0}$ simplifies into $X=$ constant.

We thus get that, when $t$ varies, the two-dimensional cells of the Voronoi diagram which are closer to $\ell_{1}(t)$ and $\ell_{2}(t)$ than to $\ell_{3}(t)$ varies continuously, with a constant topology and constant incidence relations with the trisector. As the same study may be done, replacing $\ell_{1}(t)$ and $\ell_{2}(t)$ by the other pairs of lines, this proves the theorem for all two-dimensional cells.

Finally, let $P$ be a point of the region of $\ell_{1}(t)$ (i.e., a point which is closer to $\ell_{1}(t)$ than the other lines) and $Q$ its orthogonal projection on $\ell_{1}(t)$. Then, any point of the segment $P Q$ lies also in the region of $\ell_{1}(t)$. It follows that the region of $\ell_{1}(t)$ is homeomorphic to a solid cylinder and has thus a constant topology. As this region varies continuously with $t$, as well as the two-dimensional cells of its border, this finishes the proof of the theorem.

\section{Configurations of Three Lines Whose Trisector Contains a Line}

We present here a simple geometric proof of Theorem 2, which states that the trisector of three pairwise skew lines that are not all parallel to a common plane consists of a cubic and a line if and only if the hyperboloid of one sheet containing the three skew lines is of revolution. Note that a computational proof is also given by the direct proof of the Main Lemma, in which we proved that the trisector contains a line if and only if the hyperboloid is of revolution, and by Theorem 1, which states that the trisector contains a line if and only if it is a cubic and line.

Consider three lines $\ell_{1}, \ell_{2}$, and $\ell_{3}$ whose trisector includes a line $\ell$. Any point $p$ on $\ell$ is equidistant to $\ell_{1}, \ell_{2}$, and $\ell_{3}$, so $p$ is the center of a sphere that is tangent to all of $\ell_{1}, \ell_{2}$, and $\ell_{3}$. Consider three distinct such points on $\ell$ and the three corresponding spheres. If these spheres have a common intersection, then this common intersection is a circle (possibly reduced to a point) and all lines tangent to the three spheres lie in the plane of this circle, which contradicts the general position assumption. Otherwise, the set of lines tangent to the three spheres are the ruling(s) of a single quadric of revolution with symmetry axis the line through their centers [6, Lemma 7]. Note that this quadric is a hyperboloid of one sheet since it cannot be a cone or a cylinder by the general position assumption.

Conversely, if three lines lie on a quadric of revolution, any point on the axis of revolution is equidistant to the three lines. Thus the trisector of the three lines contains a line, and, by Theorem 1, the trisector of three lines in general position is a nonsingular quartic or a cubic and a line.

\section{Algorithms}

In this section, we prove Theorem 3. We start by presenting an algorithm for determining a plane separating the two components of any two-dimensional Voronoi cell. 


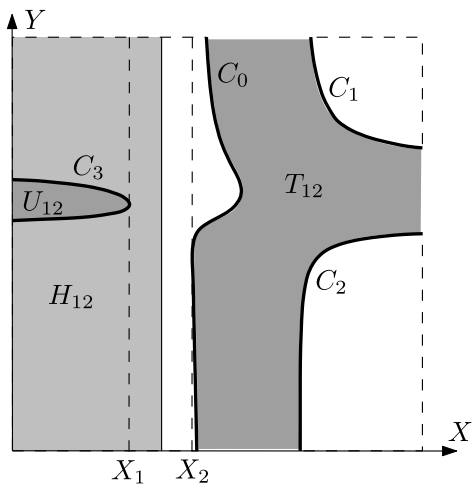

(a)

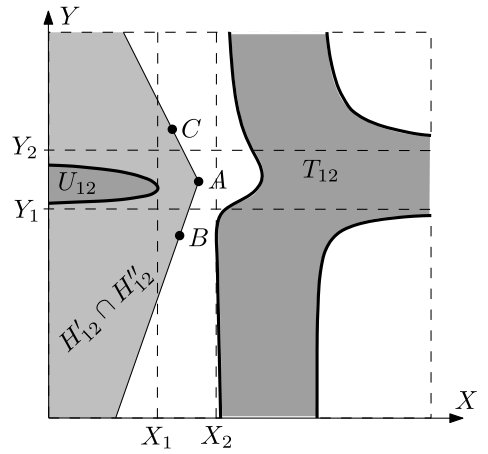

(b)

Fig. 5 Separating the two components of a two-dimensional Voronoi cell

Refer to Fig. 5(a). This plane may be nonrational; indeed, as we shall see in Proposition 21 , it is possible that no rational separating plane exists. We then show how this algorithm can be modified to produce a rational linear test for this problem when the three input lines are rational. As we will see, this algorithm leads directly to another rational linear test for separating the four connected components of the cell of dimension one. Finally, we conclude the proof of Theorem 3 by showing how points on a branch of the trisector can be ordered using a linear form with rational coefficients.

Recall that a regular point $\left(X_{0}, Y_{0}, Z_{0}\right)$ on a curve is critical with respect to the $X$-axis if the multiplicity of intersection of the curve with the plane $X=X_{0}$ is at least 2. This means that the plane $X=X_{0}$ is tangent to the curve. If the point is at infinity on an asymptote that lies in the plane $X=X_{0}$, then the curve and the plane intersect with a multiplicity that is generically equal to 2 . This is consistent with the fact that, roughly speaking, the curve is tangent to its asymptote at infinity. We say that a regular point at infinity whose asymptote belongs to the plane $X=X_{0}$ is critical if the multiplicity of intersection of the curve with the plane $X=X_{0}$ is at least 3 at this point. Note that if the multiplicity is exactly 3 , then the two branches of the curves are on the same side of the plane. The critical points that are possibly at infinity are called the generalized critical points, and the corresponding $X$-coordinates are called the generalized critical values. If $F(X, Y)=0$ is the equation of the projection of a curve $\mathcal{C}$ on the $X Y$-plane, the generalized critical values of $\mathcal{C}$ with respect to the $X$-axis are roots (in $X$ ) of the discriminant of $F$ with respect to $Y$. (This discriminant may have more roots corresponding to the singular points of the projected curve, if any.) Recall also that the asymptotes of $\mathcal{C}$ that are parallel to $Y Z$-plane but not vertical (i.e., not parallel to the $Z$-axis) lie in planes $X=X_{0}$ such that $X_{0}$ is a root (in $X$ ) of the leading coefficient of $F$ seen as a polynomial in $Y$.

\section{Determining a linear test for separating the two connected components of a two- dimensional Voronoi cell}

Input: three lines $\ell_{1}, \ell_{2}$, and $\ell_{3}$ in general position and $i \neq j \in\{1,2,3\}$.

Output: a half-space $H_{i j}$ that strictly contains $U_{i j}$ and whose complement strictly contains $T_{i j}$. 
(i) Determine a Cartesian coordinate system $(X, Y, Z)$ such that the $Z$-axis is parallel to the common perpendicular of $\ell_{i}$ and $\ell_{j}$ and such that the $X$ - and $Y$-axes are parallel to the two bisector lines, in a plane perpendicular to the $Z$-axis, of the projection of $\ell_{i}$ and $\ell_{j}$ onto that plane.

(ii) In this frame, compute all the generalized critical values of the trisector with respect to the $X$-axis. If there is no generalized critical value, exchange the $X$ - and $Y$-axes (and compute the generalized critical values with respect to the new $X$-axis).

(iii) Compute the $X$-values of the two trisector asymptotes that are parallel to the $Y Z$-plane. If the minimum of these values is smaller than or equal to the smallest generalized critical value, then change the orientation of the $X$-axis. Denote by $X_{1}$ the smallest generalized critical value (with respect to the $X$-axis) of the trisector and by $X_{2}$ the smallest of the other generalized critical values.

(iv) Pick a value $\tilde{x}$ in the open interval $\left(X_{1}, X_{2}\right)$. The half-space, $H_{i j}$, of equation $X<\tilde{x}$ contains $U_{i j}$ and the half-space $X>\tilde{x}$ contains $T_{i j}$.

Proof of correctness Assume without loss of generality that $i=1$ and $j=2$. By Proposition 18, the trisector has no critical point in the $Y$-direction after Step (ii).

First note that the asymptotes of the trisector are never vertical (i.e., parallel to the $Z$-axis) because otherwise, by Proposition 11 and since $\ell_{1}$ and $\ell_{2}$ are horizontal, the line $\ell_{3}$ would be horizontal (its direction would be the symmetric of the one of $\ell_{1}$ with respect to a vertical plane), contradicting the general position assumption.

It thus follows, since the directions of the asymptotes, projected on the $X Y$-plane, are parallel to the $X$ - or $Y$-axis (by Proposition 17), that the oriented directions of the asymptotes of the branches of the projected trisector are invariant (in the direction $\pm X$ or $\pm Y$ ) by continuous deformation on the set of triplets of lines in general position.

It thus follows from Proposition 13 and from the analysis of one configuration (see Fig. 5) that the two projected asymptotes of the branch $C_{3}$ have the same oriented direction. Thus $C_{3}$ has (at least) a critical point with respect to this direction, which is $X$ since there is no critical point with respect to the $Y$-axis. We assume, for now, that the oriented asymptotic direction of the two branches of $C_{3}$ is the $-X$ direction (as in Fig. 5), by changing, if necessary, the orientation of the axis.

In the sequel of the proof, all the critical points and values are considered generalized and with respect to the $X$-axis.

Now, the plane, denoted $\mathcal{P}$, parallel to the $Y Z$-plane through a critical point of the trisector does not intersect the trisector in any other point in $\mathbb{R}^{3}$ because the intersection at the critical point has multiplicity (at least) two, the plane intersects the trisector in two points at infinity (by Proposition 17), and the trisector has degree four (it is the intersection of two quadrics). It thus follows that $C_{3}$ has a unique critical point and that this critical point is strictly left (i.e., has smaller $X$-coordinate) of all the other critical points of the trisector. Furthermore, the plane $\mathcal{P}$ through this leftmost critical point, that is, the plane of equation $X=X_{1}$, separates (strictly, except for the critical point) the branch $C_{3}$ from the other branches and leaves $C_{3}$ on its left. In other words, the half-space $X<X_{1}$ contains $C_{3}$ except for its critical point and the half-space $X>X_{1}$ contains the other branches. It then follows from the definition of 
$X_{2}$ that, for any $\tilde{x} \in\left(X_{1}, X_{2}\right)$, the half-space $X<\tilde{x}$ contains $C_{3}$ and the half-space $X>\tilde{x}$ contains the other branches of the trisector. We thus get that the half-space $X<\tilde{x}$ contains $U_{12}$ because $U_{12}$ is bounded by $C_{3}$ (by Proposition 14) and lies on a hyperbolic paraboloid of equation $Z=\gamma X Y, \gamma \in \mathbb{R}$ (see (2)). Similarly, the halfspace $X>\tilde{x}$ contains $T_{12}$.

It remains to show that the orientation of the $X$-axis we have considered so far is the orientation obtained in Step (iii) of the algorithm. Let $X_{c}$ denote the minimum generalized critical value and $X_{a}$ the minimum $X$-value of the asymptotes parallel to the $Y Z$-plane, at the end of Step (ii). Recall that the orientation of the $X$-axis is changed in Step (iii) if and only if $X_{a} \leq X_{c}$. We show that the orientation of the $X$-axis is changed in Step (iii) if and only if the orientation is not as assumed above, that is, if the oriented asymptotic direction of the two branches of $C_{3}$ is the $+X$ direction.

We have proved that $C_{3}$ has as a unique critical value and no asymptote parallel to the $Y Z$-plane. We have also shown that if the orientation of the $X$-axis is as assumed above, then $C_{3}$ is to the left of plane $X=\tilde{x}$, which is to the left of all the other branches. Thus, $X_{c}<X_{a}$, and the orientation is not changed in Step (iii). Conversely, suppose, for a contradiction, that the orientation of the $X$-axis is not as assumed above and that $X_{c}<X_{a}$. As before, by analyzing one particular example, we have (by continuity and Proposition 13) that two of the asymptotes of the branches of $C_{1}$ and $C_{2}$ have direction $-X$ (in projection) and the two others have direction $+Y$ and $-Y$. The plane $X=X_{c}$ intersects the trisector at the generalized critical point with multiplicity (at least) two and at two other points at infinity (by Proposition 17). Furthermore, since the generalized critical point is to the left of the vertical asymptotes of $C_{1} \cup C_{2}$, the plane $X=X_{c}$ intersects the trisector somewhere else (or with higher multiplicity), which is not possible since the trisector has degree four. Hence, $X_{a} \leq X_{c}$, and the orientation of the $X$-axis is changed in Step (iii).

The algorithm requires computing the generalized critical values of the trisector with respect to the $X$ and $Y$-directions. We proved (in Proposition 18) that the trisector has no critical values in one of these directions, and the proof can easily be extended to show that the trisector has no generalized critical values in this direction. We show below that the trisector admits at most four generalized critical values with respect to the other direction. We consider below the coordinate system obtained after Step (ii) of the algorithm above.

Lemma 20 The trisector has three or four generalized critical values with respect to the $X$-direction. Moreover, the trisector has one generalized critical point on $C_{3}$, one on $C_{1} \cup C_{2}$, and either two on $C_{0}$ or $C_{0}$ is a line perpendicular to the $X$-axis.

Proof We consider here generalized critical points and values with respect to the $X$-direction. Recall that we proved in the proof of correctness of the algorithm that $C_{3}$ has exactly one critical point.

The projection on the $X Y$-plane of the trisector is a curve of degree four (since the trisector is the intersection of two quadrics). Furthermore, it has degree two in $X$ and degree two in $Y$ because, in the $X Y$-plane, the curve intersects any 
line parallel to the $X$ - or $Y$-axis in at most two points since there are two other points of intersection at infinity (by Proposition 17). The projected curve thus has the equation $A(X) Y^{2}+B(X) Y+C(X)=0$ where $A, B$, and $C$ are polynomials of degree two in $X$. Its generalized critical values are roots of the discriminant, $\Delta(X)=B(X)^{2}-4 A(X) C(X)$, of this polynomial with respect to $Y$. As this discriminant has degree four, there are either 0,2 , or 4 generalized critical values counted with multiplicity.

Now, we have that $C_{0}$ has two identical asymptotes that are perpendicular to the $X$-axis (by Propositions 14 and 17 and Step (ii) of the algorithm). Suppose first that $C_{0}$ is not entirely critical. Then, $C_{0}$ has at least two generalized critical values (the infimum and supremum values of the $X$-coordinates of its points), and this number, counted with multiplicity, is even (it is the number of local extremums of the $X$-coordinates). Thus, since $C_{3}$ has exactly one critical point, $C_{0}$ has exactly two generalized critical values and $C_{1} \cup C_{2}$ exactly one. It follows that $C_{0}$ has exactly two generalized critical points and $C_{1} \cup C_{2}$ exactly one since none of these branches is entirely critical. Indeed, $C_{0}$ is not entirely critical by assumption, and neither $C_{1}$ nor $C_{2}$ is entirely critical because they both admit an asymptote whose projection onto the $X Y$-plane is parallel to the $X$-axis. Indeed, as argued in the proof of correctness of the algorithm, the direction of each projected asymptote is invariant by continuous deformation on the set of triplets of lines in general position, and it follows from Proposition 13 and the analysis of one example that, in projection, both $C_{1}$ and $C_{2}$ admit an asymptote that is orthogonal to the one of $C_{0}$ and thus that is parallel to the $X$-axis.

Consider now the case where $C_{0}$ is entirely critical. Then, it projects on the $X Y$-plane to a line perpendicular to the $X$-axis. It is planar and thus contained in the intersection of a plane and a quadric (the bisector of any two of the input lines). $C_{0}$ is thus a line or an irreducible conic. The trisector never contains an irreducible conic (by Theorem 1), thus $C_{0}$ is a line that is perpendicular to the $X$-axis (since its projection on the $X Y$-plane is). The corresponding critical value is, by continuity, a double root of the discriminant $\Delta(X)$. Thus, $C 1 \cup C 2$ has, as before, exactly one generalized critical point.

The following proposition shows that the separating plane computed in the above algorithm may be nonrational.

Proposition 21 There exist three rational lines for which the two connected components of any two-dimensional Voronoi cell cannot be separated by a rational plane.

Proof Let $\mathcal{P}$ denote any plane separating $U_{i j}$ and $T_{i j}$. Since $\mathcal{P}$ does not intersect $C_{0}$, it is necessarily parallel to the asymptote of $C_{0}$ (see Proposition 14).

We now exhibit an example of three rational lines such that there exists no rational plane parallel to an asymptote of their trisector, which will conclude the proof. Consider three lines $\ell_{1}, \ell_{2}$, and $\ell_{3}$ in general position that have directions $(1,0,0)$, $(1,1,0)$, and $(2,0,1)$, respectively. By Proposition 11 , the four asymptotes of their trisector are parallel to the four trisector lines of three concurrent lines (say, through the origin) with directions those of $\ell_{1}, \ell_{2}$, and $\ell_{3}$; let $\ell_{1}^{\prime}, \ell_{2}^{\prime}$, and $\ell_{3}^{\prime}$ denote these lines. 
The pair of bisector planes of $\ell_{1}^{\prime}$ and $\ell_{2}^{\prime}$ has a square root of 2 in its coefficient: its equation (see (1)) factors into $(X-(1+\sqrt{2}) Y)(X-(1-\sqrt{2}) Y)$, which is the equation of a pair of conjugate planes over $\mathbb{Q}(\sqrt{2})$ (the field extension of $\mathbb{Q}$ by $\sqrt{2}$ ). Similarly, the bisector planes of $\ell_{1}^{\prime}$ and $\ell_{3}^{\prime}$ is a pair of conjugate planes over $\mathbb{Q}(\sqrt{5})$ (it has the equation $(X-(2+\sqrt{5}) Z)(X-(2-\sqrt{5}) Z))$. It follows that the four lines of intersection of these two pairs of planes are conjugate over $\mathbb{Q}(\sqrt{2}, \sqrt{5})$.

Furthermore, these four lines are not all parallel to a common plane because the intersection of the two planes that are conjugate over $\mathbb{Q}(\sqrt{2})$ is the $Z$-axis, which properly intersects each of the two other conjugate planes; thus, on each of these latter conjugate planes, the two lines of intersection properly intersect, and thus any plane parallel to them is parallel to the plane they define; since the two conjugate planes are not coplanar, no plane is parallel to the four lines of intersection.

Now, any rational plane that is parallel to one of these four lines is also parallel to the three others (since a rational plane is invariant by conjugation over $\mathbb{Q}(\sqrt{2}, \sqrt{5})$ ). Since this is not possible, there is no rational plane that is parallel to the asymptote of $C_{0}$, which concludes the proof.

We now present an algorithm for determining a rational linear test for separating the two components of any two-dimensional Voronoi cell of three rational lines. Refer to Fig. 5(b).

\section{Determining a rational linear test for separating the two connected components of a two-dimensional Voronoi cell}

Input: three rational lines $\ell_{1}, \ell_{2}$, and $\ell_{3}$ in general position in a coordinate system $(\tilde{X}, \tilde{Y}, \tilde{Z})$ and $i \neq j \in\{1,2,3\}$.

Output: two rational half-spaces $H_{i j}^{\prime}$ and $H_{i j}^{\prime \prime}$ such that $H_{i j}^{\prime} \cap H_{i j}^{\prime \prime}$ strictly contains $U_{i j}$ and its complement strictly contains $T_{i j}$.

(i-iii) Idem as in the previous algorithm.

(iv) Compute the two $Y$-values of the two trisector asymptotes that are parallel to the $X Z$-plane. Let $Y_{1}<Y_{2}$ denote these two values.

(v) Determine a point $A$ with rational coordinates in the original $(\tilde{X}, \tilde{Y}, \tilde{Z})$-frame such that its $X$-, $Y$-, and $Z$-coordinates in the $(X, Y, Z)$ frame are in $\left(X_{1}, X_{2}\right)$, in $\left(Y_{1}, Y_{2}\right)$, and equal to 0 , respectively; let $X_{A}$ denote its $X$-coordinate in the $(X, Y, Z)$ frame.

(vi) Determine two points $B$ and $C$ with rational coordinates in the original $(\tilde{X}, \tilde{Y}, \tilde{Z})$-frame such that their $X-, Y$-, and $Z$-coordinates in the $(X, Y, Z)$ frame are, for $B$, in $\left(X_{1}, X_{A}\right)$, in $\left(-\infty, Y_{1}\right)$, and equal to 0 , respectively, and for $C$, in $\left(X_{1}, X_{A}\right)$, in $\left(Y_{2},+\infty\right)$, and equal to 0 , respectively.

(vii) Let $P_{i j}$ (resp. $P_{i j}^{\prime}$ ) be the plane through $A$ and $B$ (resp. $C$ ) that is parallel to the $Z$-axis. Let $H_{i j}^{\prime}$ (resp. $H_{i j}^{\prime \prime}$ ) be the open half-space bounded plane $P_{i j}$ (resp. $P_{i j}^{\prime}$ ) that contains the point at infinity in the $-X$-direction.

Remark 22 Note that the transformation from the $(\tilde{X}, \tilde{Y}, \tilde{Z})$-frame to the $(X, Y, Z)$ frame is not necessarily rational since the $X$ - and $Y$-axes are not necessarily rational in the $(\tilde{X}, \tilde{Y}, \tilde{Z})$-frame. Hence, the plane through $A$ and parallel to the $Y$ - and $Z$-axes 
is not necessarily rational in the $(\tilde{X}, \tilde{Y}, \tilde{Z})$-frame. Nonetheless, the rational coordinates of the points $A, B$, and $C$ can easily be computed, using interval arithmetic (see the proof of correctness for details).

Proof of correctness We assume without loss of generality that $i$ and $j$ are equal to 1 and 2, respectively. Notice first that point $A$ (and similarly for $B$ and $C$ ) can easily be chosen with rational coordinates in the original $(\tilde{X}, \tilde{Y}, \tilde{Z})$-frame using interval arithmetic. Indeed, the generalized critical values $X_{1}$ and $X_{2}$ in the local $(X, Y, Z)$-frame are the solutions of a polynomial with not necessarily rational coefficients (because the transformation from the original frame to the local frame is not necessarily rational). The $X$-coordinate $X_{A}$ of $A$ in the $(X, Y, Z)$-frame can be chosen anywhere in between $X_{1}$ and $X_{2}$. Thus, isolating the roots of this polynomial with interval arithmetic (see, e.g., [9]) gives a feasible interval for $X_{A}$, in between the intervals for $X_{1}$ and $X_{2}$. Similarly for the $Y$-coordinate of $A$. Transforming the resulting $X Y$-box back to the original $(\tilde{X}, \tilde{Y}, \tilde{Z})$-frame yields, with interval arithmetic, another nonempty box from which the point $A$ can be chosen with rational coordinates in the original frame.

Now, we have seen in the proof of correctness of the previous algorithm that the component $C_{3}$ has exactly one critical value with respect to the $X$-axis, no critical value with respect to the $Y$-axis, and two asymptotes in the $-Y$-direction. The component $C_{3}$ is thus contained in the region defined by $X<X_{1}$ and $Y_{1}<Y<Y_{2}$. It follows that $H_{i j}^{\prime} \cap H_{i j}^{\prime \prime}$ contains $U_{i j}$.

On the other hand, the complement of $H_{i j}^{\prime} \cap H_{i j}^{\prime \prime}$ strictly contains $T_{i j}$ because for any value $\tilde{x} \in\left(X_{A}, X_{2}\right)$, the half-space $X>\tilde{x}$ contains $T_{i j}$ (as proved above), and this half-space is contained in the complement of $H_{i j}^{\prime} \cap H_{i j}^{\prime \prime}$.

Finally, the plane $P_{i j}$ is rational since $A$ and $B$ are rational as well as the $Z$-axis (since it is the common perpendicular to $\ell_{i}$ and $\ell_{j}$ ). Similarly, plane $P_{i j}^{\prime}$ is also rational.

Remark 23 Note that, if the three input lines are not rational, the above algorithm remains valid except for the fact that the output half-spaces are not necessarily rational anymore (since the common perpendicular to $\ell_{i}$ and $\ell_{j}$ is not necessarily rational).

Separation of the Four Connected Components of the Trisector of Three Lines Consider three lines $\ell_{1}, \ell_{2}$, and $\ell_{3}$ and the half-spaces $H_{i j}^{\prime}$ and $H_{i j}^{\prime \prime}$ obtained by the above algorithm. Proposition 14 (and Remark 23) directly yields the following result.

Proposition 24 For any point $p$ on the trisector of $\ell_{1}, \ell_{2}$, and $\ell_{3}$, if $p$ belongs to both half-spaces $H_{i j}^{\prime}$ and $H_{i j}^{\prime \prime}$ for some $i \neq j \in\{1,2,3\}$, then $p$ lies on $C_{k}$ (with $k \in\{1,2,3\}$ distinct from $i$ and $j$ ), otherwise $p$ lies on $C_{0}$. Furthermore, if the three input lines are rational, the coefficients of $H_{i j}^{\prime}$ and $H_{i j}^{\prime \prime}$ are rational.

Determining a Linear Test for Ordering Points on a Branch of the Trisector Every branch of the trisector of three lines is monotonic in the $Y$-direction computed in Steps (i-ii) of the above algorithms (see the proof of correctness of the first algorithm). This gives a linear test for ordering points on each branch of the trisector. 
It remains to show that, if the three input lines are rational, we can determine a rational direction in which every branch of the trisector is monotonic, yielding a rational linear test for ordering points on each trisector.

\section{Determining a rational linear test for ordering points on a branch $\mathbf{C}_{k}$ of the trisector}

Input: three rational lines $\ell_{1}, \ell_{2}$, and $\ell_{3}$ in general position and $k \in\{0,1,2,3\}$.

Output: a rational direction in which $C_{k}$ is strictly monotonic.

(0) Choose $i \neq j \in\{1,2,3\}$ such that $\{i, j, k\} \neq\{1,2,3\}$ and initialize $\alpha$ to a small positive value.

(i-ii) Idem as in the previous algorithms. If the $Y$-axis is rational in the original coordinate system, output it.

(iii) Rotate clockwise the $Y$-axis about the $Z$-axis by an angle of at most $\alpha$ and such that the resulting $Y^{\prime}$-axis is rational in the original coordinate system.

(iv) Compute the critical points of the trisector with respect to the $Y^{\prime}$-axis and determine (see Proposition 24) if any critical point lies on $C_{k}$. If so, divide $\alpha$ by two and recurse Step (iii) after changing the clockwise/counterclockwise orientation of the rotation. Otherwise, output the $Y^{\prime}$-axis.

Proof of correctness We can assume without loss of generality that, after Step 0, $i, j=1,2$ and $k \neq 3$. As argued before, the coordinate system computed in Step (ii) is such that $C_{0}, C_{1}, C_{2}$ are strictly monotonic in the $Y$-direction. Furthermore, in projection onto the $X Y$-plane, $C_{0}$ has two (identical) asymptotes parallel to the $Y$-axis, and $C_{1}$ and $C_{2}$ both have one asymptote parallel to the $X$-axis and another parallel to the $Y$-axis (by Propositions 13, 14, and 17 and from the analysis of one configuration). Hence, each of these branches is also strictly monotonic in any direction obtained by a sufficiently small rotation of the $Y$-axis about the $Z$-axis in the clockwise or counterclockwise direction (depending on the branch).

Remark 25 The orientation, clockwise or counterclockwise, of the rotation in Step (iii) can also be determined as follows. Compute the two $X$-values of the two trisector asymptotes that are parallel to the $Y Z$-plane and choose a strictly larger value $X_{0}$. Compute the two points of intersection of the trisector with the plane $X=X_{0}$; let $P_{+}$ denote the one with larger $Y$-coordinate and $P_{-}$the other one. Determine whether $P_{+}$or $P_{-}$lies on the branch $C_{k}$. If $P_{+}$lies on $C_{k}$, always rotate the $Y$-axis counterclockwise in Step (iii) ( $C_{k}$ is the branch $C_{1}$ in Fig. 5(a)). If $P_{-}$lies on $C_{k}$, rotate the $Y$-axis clockwise ( $C_{k}$ is the branch $C_{2}$ in Fig. 5(a)). Otherwise, both directions of rotation will work ( $C_{k}$ is the branch $C_{0}$ in Fig. 5(a)).

We presented, in this section, algorithms for determining linear tests for separating the connected components of the Voronoi cells of dimensions one and two and for ordering points on any branch of the trisector. Moreover, we showed how to determine rational linear tests for these problems in the case where the input lines are rational. This concludes the proof of Theorem 3. 


\section{Conclusion}

We presented a complete description of the Voronoi diagram of three lines that are pairwise skew and not all parallel to a common plane. We also presented linear tests for answering queries of the form, given a point in a one or two-dimensional Voronoi cell, determine in which of its connected components it lies. Finally, we showed how points on a branch of the trisector can be ordered using linear forms. Moreover, these linear forms have rational coefficients if the input lines are rational.

Future work includes the characterization of the topology of the Voronoi diagram of three lines that are not in general position. Note that, in this case, the topology of the Voronoi diagram does indeed change; for instance, when three pairwise skew lines are all parallel to a common plane, their bisectors are hyperbolic paraboloids of the form $Z=F_{i j}(X, Y)$, and it follows that their trisector consists of two branches (instead of four) as it is the intersection of one of the bisectors with a hyperbolic cylinder whose axis is parallel to the $Z$-axis (and whose equation is $F_{12}(X, Y)-$ $\left.F_{13}(X, Y)=0\right)$. Note also that when two of the lines are coplanar, their bisector is one or two planes, and the trisector is thus either the intersection of two such bisectors or the intersection of one such bisector with a hyperbolic paraboloid.

Another problem is to study Voronoi diagrams of up to six lines; indeed, designing an incremental algorithm for computing the Voronoi diagrams of $n$ lines would require a thorough understanding of the case of six lines since the arcs of the diagram are defined by five lines (three for the trisector and one additional line for each endpoint). Finally, the two major problems remain the determination of the complexity of Voronoi diagrams of $n$ lines and the design of efficient algorithms for computing Voronoi diagrams of lines, segments, triangles, or polyhedra.

Acknowledgements We would like to thank C. Hillar and C. Borcea for useful discussions.

\section{Appendix: Maple-Sheet Computations}

Table 2 About the proof of the Main Lemma

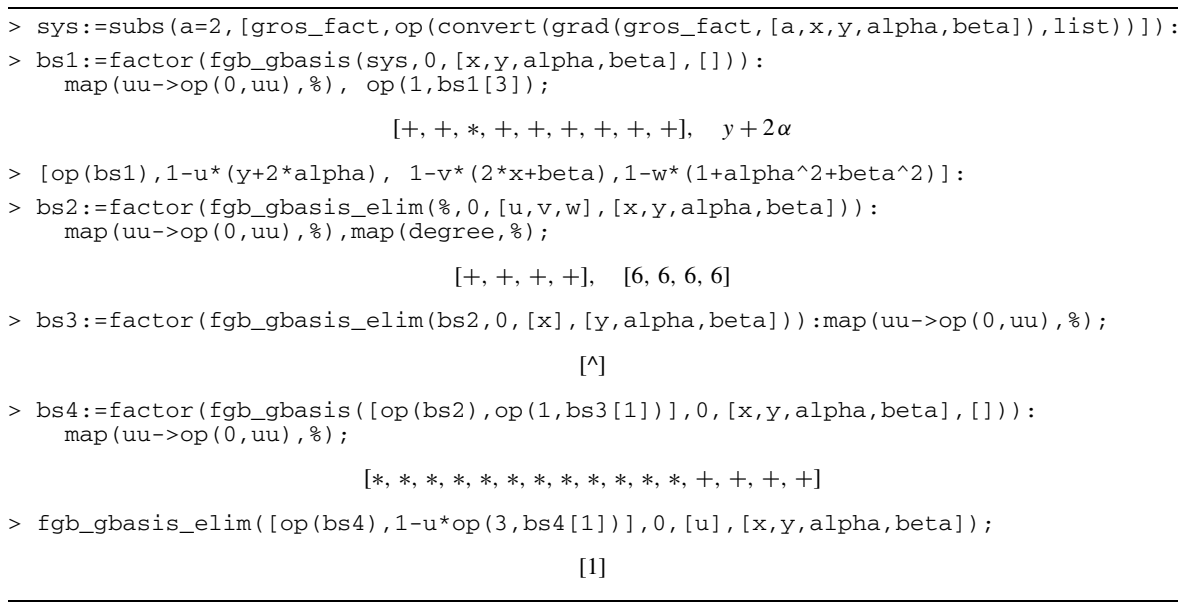




\section{Table 2 (Continued)}

$>$ bs5:=factor (fgb_gbasis ([op (bs4), op (3,bs4[1])],0, [x,y,alpha, beta], [] )) : $\operatorname{map}(u u->o p(0, u u), \%)$;

$$
[+,+,+,+,+, *,+,+,+,+,+,+, *,+,+, *, *, *, *, *, *, *, *,+,+,+,+]
$$

> fgb_gbasis_elim([op (bs5),1-u*op (3,bs5[6] )], 0, [u], [x,y, alpha, beta]);

$>$ bs6:=factor (fgb_gbasis ([op (bs5), op (3, bs5[6]) ], 0, [x,y, alpha, beta ], [] ) ) : map (uu->op $(0, u u), \%$;

$$
[+,+,+,+,+,+,+,+,+,+,+,+,+,+,+,+]
$$

$>$ bs7:=factor (fgb_gbasis_elim(bs6,0,[y], [x, alpha, beta $])): \operatorname{map}(u u->o p(0, u u), \%)$;

$$
[*, *, *, *, *, *, *, *, *, *, *, *, *, *, *, *, *, *, *, *, *, *, *, *, *, *, \wedge]
$$

$>$ bs $8:=$ factor (fgb_gbasis $([\mathrm{op}(\mathrm{bs} 6)$, op (1,bs7 [nops (bs7) ]) ], 0, [x,y, alpha, beta ], [ ] )): $\operatorname{map}(u u->o p(0, u u), \%)$;

$$
[+,+,+,+,+,+,+,+,+,+, \ldots,+,+,+,+,+,+,+,+,+,+]
$$

$>\operatorname{bs} 9:=$ factor $\left(f g b \_g b a s i s \_e l i m(b s 8,0,[a l p h a],[x, y, b e t a])\right): \operatorname{map}(u u->o p(0, u u), \%)$;

$$
[*, *, *, *, *, *, *, *, *, *, \ldots, *, *, *, *, *, *, *, *, *, *]
$$

> fgb_gbasis_elim([op(bs9),1-u*op (nops (bs9[1]),bs9[1])],0,[u],[x,y, alpha, beta]) ;

$>$ bs10:=factor (fgb_gbasis ([op (bs8), op (nops (bs9[1]), bs9[1])],0,

$[\mathrm{x}, \mathrm{y}, \mathrm{alpha}, \mathrm{beta}],[]))$ :

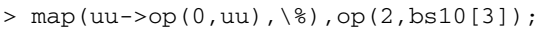

$$
[+,+, *,+,+,+,+,+,+, *,+,+,+,+,+, \ldots,+,+,+,+,+], \quad y+2 \alpha
$$

$>\left[o p(b s 10), 1-u *(1+a l p h a \wedge 2+b e t a \wedge 2), 1-v^{*}(y+2 * a l p h a), 1-w^{*}(2 * x+b e t a)\right]:$

$>$ bs11:=factor (fgb_gbasis_elim(\%, 0, [u,v,w], [x,y,alpha, beta $]))$ : $\operatorname{map}(u u->o p(0, u u), \%)$;

$$
[+,+,+, *,+,+,+,+,+,+,+,+,+,+,+]
$$

> fgb_gbasis_elim([op (bs11),1-u*op (2, bs11[4])],0,[u],[x,y,alpha, beta ]) ;

\section{[1]}

$>$ bs12:=factor (fgb_gbasis ([op (bs11), op (2,bs11[4])],0,[x,y,alpha, beta], [] )): $\operatorname{map}(u u->o p(0, u u), \%$, map (degree, $\%$ );

$$
[+,+,+], \quad[4,4,4]
$$

$>\operatorname{bs} 12[3] ;$

$>$ Gamma $:=\left(4 * y^{*} \text { alpha }-4 * x * \text { beta }-3\right)^{\wedge} 2+3 *(2 * x+\text { beta })^{\wedge} 2+12 *(y+2 * \text { alpha })^{\wedge} 2+75$;

> simplify (Gamma2-bs12[3]);

$$
\begin{gathered}
16 \alpha^{2} y^{2}+84-32 \beta x \alpha y+16 \beta^{2} x^{2}+12 x^{2}+12 y^{2}+24 y \alpha+48 \alpha^{2}+36 \beta x+3 \beta^{2} \\
\Gamma 2:=(4 y \alpha-4 \beta x-3)^{2}+3(2 x+\beta)^{2}+12(y+2 \alpha)^{2}+75
\end{gathered}
$$

$>$ [op (sys), 1-u* (1+alpha^2+beta^2),1-v* $(y+2 * a l p h a), 1-w^{*}(2 * x+$ beta $), 1-t *$ Gamma2 ]

$>$ fgb_gbasis $(\%, 0,[u, v, w, t],[x, y, a l p h a, b e t a])$; 
Table 3 For the proof of Lemma 8

$>$ factor(subs $\left.\left(y=-a * a l p h a, b i g \_f a c t\right)\right)$;

$$
\begin{aligned}
& \left(\alpha^{4} a^{4}+2 \beta x \alpha^{2} a^{3}+x^{2} a^{2}+\beta^{2} x^{2} a^{2}-2 a^{2} \alpha^{2}+1+\beta^{2}\right) \\
& \left(\beta^{2}-4 a^{2}-4 a^{2} \alpha^{2}-4 a^{4}-4 a^{4} \alpha^{2}-2 a \beta x-4 \beta x a^{3}+x^{2} a^{2}\right)^{2}
\end{aligned}
$$

$>\mathrm{f} 0:=\operatorname{collect}(\mathrm{op}(1, \%), \mathrm{x}) ; \mathrm{fl}:=\operatorname{collect}(\mathrm{op}(1, \mathrm{op}(2, \% \%)), \mathrm{x})$;

$$
\begin{aligned}
& f 0:=\left(a^{2} \beta^{2}+a^{2}\right) x^{2}+2 \beta x \alpha^{2} a^{3}+\alpha^{4} a^{4}+1+\beta^{2}-2 a^{2} \alpha^{2} \\
& f 1:=x^{2} a^{2}+\left(-2 a \beta-4 \beta a^{3}\right) x+\beta^{2}-4 a^{2}-4 a^{2} \alpha^{2}-4 a^{4}-4 a^{4} \alpha^{2}
\end{aligned}
$$

$>$ factor $\left(\operatorname{subs}\left(x=-\right.\right.$ beta $\left.\left./ a, b i g \_f a c t\right)\right)$;

$$
\begin{aligned}
& \left(\beta^{4}-2 a^{2} \beta^{2}+a^{4}+a^{4} \alpha^{2}+2 \beta^{2} \alpha a y+\alpha^{2} y^{2} a^{2}+y^{2} a^{2}\right) \\
& \left(4+4 \beta^{2}+4 a^{2}+4 a^{2} \beta^{2}-a^{4} \alpha^{2}+4 a y \alpha+2 y a^{3} \alpha-y^{2} a^{2}\right)^{2}
\end{aligned}
$$

$>\mathrm{g} 0:=\operatorname{collect}(\mathrm{op}(1, \%), \mathrm{y}) ; \mathrm{g} 1:=\operatorname{collect}(\mathrm{op}(1, \mathrm{op}(2, \%))), \mathrm{y})$;

$$
\begin{aligned}
& g 0:=\left(a^{2} \alpha^{2}+a^{2}\right) y^{2}+2 \beta^{2} \alpha a y+\beta^{4}-2 a^{2} \beta^{2}+a^{4}+a^{4} \alpha^{2} \\
& g 1:=-y^{2} a^{2}+\left(4 a \alpha+2 a^{3} \alpha\right) y+4+4 \beta^{2}+4 a^{2}+4 a^{2} \beta^{2}-a^{4} \alpha^{2}
\end{aligned}
$$

Solutions of $f 1=0$ in $x$ and of $g 1=0$ in $y$ :

$>\operatorname{map}(u u->$ factor $(u u),[$ solve $(f 1, x)])$;

$$
\left[\frac{2 a^{2} \beta+\beta+2 \sqrt{a^{2}\left(a^{2}+1\right)\left(\beta^{2}+1+\alpha^{2}\right)}}{a}, \frac{2 a^{2} \beta+\beta-2 \sqrt{a^{2}\left(a^{2}+1\right)\left(\beta^{2}+1+\alpha^{2}\right)}}{a}\right]
$$

$>\operatorname{map}(u u->$ factor $(u u),[\operatorname{solve}(g 1, y)])$;

$$
\left[\frac{\alpha a^{2}+2 \alpha+2 \sqrt{\left(a^{2}+1\right)\left(\beta^{2}+1+\alpha^{2}\right)}}{a}, \frac{\alpha a^{2}+2 \alpha-2 \sqrt{\left(a^{2}+1\right)\left(\beta^{2}+1+\alpha^{2}\right)}}{a}\right]
$$

$f 0$ is a sum of square:

$>(a \wedge 2 * a l p h a \wedge 2-1+a * \text { beta*x })^{\wedge} 2+(a * x+\text { beta })^{\wedge} 2$;

$>\operatorname{simplify}(\mathrm{f} 0-\%)$;

$$
\left(a^{2} \alpha^{2}-1+a \beta x\right)^{2}+(x a+\beta)^{2}
$$

0

$a^{*} x+$ beta and $g 1$ are in the ideal generated by $y+a *$ alpha, $x * a+$ beta, and $a^{\wedge} 2 *$ alpha^2-1+a*beta* $x$ :

$>\operatorname{gbasis}\left(\left[y+a * a l p h a, x^{*} a+b e t a, a^{\wedge} 2 * a l p h a \wedge 2-1+a * b e t a * x\right], \operatorname{DRL}([a, x, y, a l p h a, b e t a])\right)$ :

$>\operatorname{normalf}\left(a^{*} x+\right.$ beta, $\left.\%\right), \operatorname{normalf}(g 1, \%)$;

$g 0$ is a sum of square:

$>\left(a * y^{\star} a l p h a+b e t a \wedge 2-a \wedge 2\right)^{\wedge} 2+a \wedge 2 *(y+a * a l p h a) \wedge 2$;

$>\operatorname{simplify}(\mathrm{g} 0-\%)$;

$$
\left(a y \alpha+\beta^{2}-a^{2}\right)^{2}+a^{2}(y+a \alpha)^{2}
$$

0

$y+a^{*}$ alpha and $f 1$ are in the ideal generated by $x^{*} a+$ beta, $y+a^{*}$ alpha, and $a^{\wedge} 2 *$ alpha^2-1+a*beta*x:

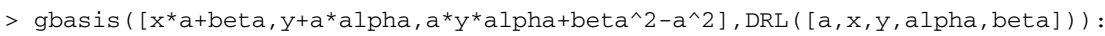

$>\operatorname{normalf}(\mathrm{y}+\mathrm{a} * a l p h a, \%), \operatorname{normalf}(\mathrm{f} 1, \%)$;

0,0 
Table 4 For the proof of Lemma 10

$>\operatorname{comp} 1:=[y=-a * a l p h a, x=$

$>(2 *$ beta*a^ $2+$ beta $) / a+2 * \operatorname{sqrt}\left((\right.$ beta^ $\left.\left.2+1+a l p h a \wedge 2) *\left(1+a^{\wedge} 2\right)\right)\right]$

$$
\operatorname{comp} 1:=\left[y=-\alpha a, x=\frac{2 \beta a^{2}+\beta}{a}+2 \sqrt{\left(1+\alpha^{2}+\beta^{2}\right)\left(1+a^{2}\right)}\right]
$$

We prove that the characteristic equation has no real root on this component.

$>$ factor (subs (comp1, Char_eq)) ;

$>$ irrat: $=$ op $(2, \%)$ :

$$
\begin{aligned}
& a^{2}\left(4-4 \beta^{2} \lambda^{3}+8 a^{2}-4 \lambda^{3}+\lambda^{4}-8 \lambda-16 \alpha^{2} \lambda a^{2}-8 \beta^{2} \lambda a^{2}+8 \alpha^{2}+4 \beta^{2}+12 a^{2} \alpha^{2}+12 a^{2} \beta^{2}\right. \\
& +4 a^{4}+8 a^{4} \beta^{2}+4 a^{4} \alpha^{2}-8 \lambda a^{2}-16 \alpha^{2} \lambda-8 \beta^{2} \lambda+8 \lambda^{2}+4 \lambda^{2} a^{2}+8 a^{2} \alpha^{2} \lambda^{2}+4 \beta^{2} \lambda^{2} a^{2} \\
& \quad+8 \beta^{2} \lambda^{2}-8 \beta \alpha a^{3} \lambda-8 \beta \alpha \lambda a+8 \beta \alpha a^{3}+8 a \beta \alpha+8 \alpha \sqrt{\% 1}-8 \lambda a^{2} \alpha \sqrt{\% 1}+\lambda^{4} \beta^{2} \\
& \quad+\lambda^{4} \alpha^{2}+4 \lambda^{2} \beta \sqrt{\% 1} a-8 \lambda \beta \sqrt{\% 1} a+12 \alpha^{2} \lambda^{2}-4 \alpha^{2} \lambda^{3}+8 \beta \sqrt{\% 1} a+8 a^{2} \alpha \sqrt{\% 1} \\
& \left.+8 \beta a^{3} \sqrt{\% 1}-4 \lambda^{3} \alpha \sqrt{\% 1}+12 \lambda^{2} \alpha \sqrt{\% 1}-16 \lambda \alpha \sqrt{\% 1}\right) \\
& \% 1:=\left(\beta^{2}+1+\alpha^{2}\right)\left(1+a^{2}\right)
\end{aligned}
$$

Consider the product of the characteristic polynomial with its algebraic conjugate:

$>\mathrm{T}:=\operatorname{expand}\left(\operatorname{irrat}{ }^{*} \operatorname{subs}\left(\operatorname{sqrt}\left(\left(1+\mathrm{a}^{\wedge} 2\right) *(\mathrm{alpha})^{\wedge}+\mathrm{beta}^{\wedge} 2+1\right)\right)=-\operatorname{sqrt}\left(\left(1+\mathrm{a}^{\wedge} 2\right) *(\mathrm{alpha} 2\right.\right.$

$>+$ beta^$\left.^{\wedge} 2+1\right)$ ), irrat)):

The real semi-algebraic set defined by $\mathrm{T}-1 / 2<0$ is empty:

$>$ sampling_negative (T-1/2, [a, alpha, beta, lambda ]);

Pre-process...............

Computing critical values of a polynomial mapping from $\mathrm{C}^{\wedge} 4$ to $\mathrm{C}$

Computing asymptotic critical values of a polynomial mapping from $\mathrm{C}^{\wedge} 4$ to $\mathrm{C}$

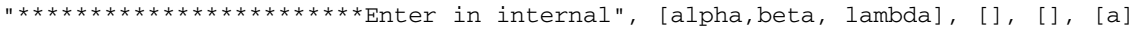
End of pre-process.............

Computing sampling points in a real hypersurface

Computing Critical Points using FGb (projection on a)

Computing Asymptotic Critical Values of a restricted to a hypersurface

Computing Critical points using FGb (projection on alpha)

Computing Asymptotic Critical Values of alpha restricted to a hypersurface

Computing Asymptotic Critical Values of alpha restricted to a hypersurface

Computing Critical Points using FGb (projection on beta)

Computing Asymptotic Critical Values of beta restricted to a hypersurface

Computing Critical Points using FGb (projection on lambda)

Isolating real solutions of a zero-dimensional system using RS

Isolating real solutions of a zero-dimensional system using RS

Isolating real solutions of a zero-dimensional system using RS

Isolating real solutions of a zero-dimensional system using RS

\section{[]}

Consider all the $3 \times 3$ minors of the matrix $P(\lambda)$ of the pencil:

$>1$ det: $=$ NULL:

$>$ for $i$ to 4 do for $j$ from $i$ to 4 do

$>1$ det: $=1$ det, $\operatorname{det}(\operatorname{minor}(P, i, j))$ :

$>$ od od:

The rank of $P(\lambda)$ is always 3 or 4 since there is no common zeros of the minors:

$>[$ ldet, $1-t *(1+a 1 p h a \wedge 2+$ beta^ 2$) *(1+a \wedge 2) *(-$ beta $+y+a * x-a * a l p h a) *$

(-beta-y+a*x+a*alpha) ] :

> fgb_gbasis_elim(\%,0,[],[t,a, x,y,alpha, beta, lambda]) ;

[1] 


\section{References}

1. Appel, K., Haken, W., Koch, J.: Every planar map is four colorable. Parts I and II. Ill. J. Math. 21, 429-567 (1977)

2. Aurenhammer, F., Klein, R.: Voronoi diagrams. In: Sack, J.R., Urrutia, J. (eds.) Handbook of Computational Geometry, Chap. 5, pp. 201-290. Elsevier, Amsterdam (2000)

3. Berberich, E., Hemmer, M., Kettner, L., Schömer, E., Wolpert, N.: An exact, complete and efficient implementation for computing planar maps of quadric intersection curves. In: Proceedings of the $21 \mathrm{st}$ ACM Annual Symposium on Computational Geometry (SoCG’05), pp. 99-115 (2005)

4. Boissonnat, J.-D., Devillers, O., Pion, S., Teillaud, M., Yvinec, M.: Triangulations in CGAL. Comput. Geom. Theory Appl. 22, 5-19 (2002)

5. Boissonnat, J.-D., Wormser, C., Yvinec, M.: Curved Voronoi diagrams. In: Boissonnat, J.-D., Teillaud, M. (eds.) Effective Computational Geometry for Curves and Surfaces. Mathematics and Visualization, pp. 67-116. Springer, Berlin (2006)

6. Borcea, C., Goaoc, X., Lazard, S., Petitjean, S.: Common tangents to spheres in $\mathbb{R}^{3}$. Discrete Comput. Geom. 35(2), 287-300 (2006)

7. Cheng, J., Lazard, S., Peñaranda, L., Pouget, M., Rouillier, F., Tsigaridas, E.: On the topology of planar algebraic curves. In: Proceedings of the 25th ACM Annual Symposium on Computational Geometry (SoCG'09) (2009)

8. Chou, S.-C., Gao, X.-S., Zhang, J.-Z.: Machine Proofs in Geometry: Automated Production of Readable Proofs for Geometry Theorems. World Scientific, Singapore (1994)

9. Collins, G.E., Johnson, J.R., Krandick, W.: Interval arithmetic in cylindrical algebraic decomposition. J. Symb. Comput. 34(2), 145-157 (2002)

10. Cox, D., Little, J., O'Shea, D.: Using Algebraic Geometry, 2nd edn. Graduate Texts in Mathematics, vol. 185. Springer, New York (2005)

11. Culver, T.: Computing the medial axis of a polyhedron reliably and efficiently. Ph.D. thesis, University of North Carolina at Chapel Hill (2000)

12. Dey, T.K., Zhao, W.: Approximate medial axis as a Voronoi subcomplex. In: SMA '02: Proceedings of the Seventh ACM Symposium on Solid Modeling and Applications, pp. 356-366. ACM, New York (2002)

13. Dupont, L., Lazard, D., Lazard, S., Petitjean, S.: Near-optimal parameterization of the intersection of quadrics: I. The generic algorithm. J. Symb. Comput. 43(3), 168-191 (2008)

14. Dupont, L., Lazard, D., Lazard, S., Petitjean, S.: Near-optimal parameterization of the intersection of quadrics: II. A classification of pencils. J. Symb. Comput. 43(3), 192-215 (2008)

15. Dupont, L., Lazard, D., Lazard, S., Petitjean, S.: Near-optimal parameterization of the intersection of quadrics: III. Parameterizing singular intersections. J. Symb. Comput. 43(3), 216-232 (2008)

16. Emiris, I., Tsigaridas, E., Tzoumas, G.: Predicates for the exact Voronoi diagram of ellipses under the Euclidean metric. Int. J. Comput. Geom. Appl. 18(6), 567-597 (2008) (Special Issue on SoCG'06)

17. Etzion, M., Rappoport, A.: Computing Voronoi skeletons of a 3-d polyhedron by space subdivision. Comput. Geom. Theory Appl. 21(3), 87-120 (2002)

18. Everett, H., Lazard, D., Lazard, S., Safey El Din, M.: The Voronoi diagram of three lines in $\mathbb{R}^{3}$. In: Proceedings of the 23rd ACM Annual Symposium on Computational Geometry (SoCG'07), S. Korea, pp. 255-264 (2007)

19. Faugère, J.-C.: FGb-A software for computing Gröbner bases. http://fgbrs.lip6.fr

20. Fortune, S.: Voronoi diagrams and Delaunay triangulations. In: Handbook of Discrete and Computational Geometry, pp. 377-388. CRC, Boca Raton (1997)

21. Hoff, K., Culver, T., Keyser, J., Lin, M., Manocha, D.: Fast computation of generalized Voronoi diagrams using graphics hardware. Comput. Graph. 33, 277-286 (1999) (Proceedings of ACM SIGGRAPH 1999, Annual Conference Series)

22. Karavelas, M.I.: A robust and efficient implementation for the segment Voronoi diagram. In: International Symposium on Voronoi Diagrams in Science and Engineering, pp. 51-62 (2004)

23. Keyser, J., Krishnan, S., Manocha, D.: Efficient and accurate B-Rep generation of low degree sculptured solids using exact arithmetic: I Representations, II Computation. Comput. Aided Geom. Des. 16(9), 841-859, 861-882 (1999)

24. Koltun, V., Sharir, M.: Three dimensional Euclidean Voronoi diagrams of lines with a fixed number of orientations. SIAM J. Comput. 32(3), 616-642 (2003)

25. Kurdyka, K., Orro, P., Simon, S.: Semialgebraic Sard theorem for generalized critical values. J. Differ. Geom. 56, 67-92 (2000) 
26. LaValle, S.M.: Planning Algorithms. Cambridge University Press, Cambridge (2006) (Also available at http://planning.cs.uiuc.edu/)

27. Lax, P.: On the discriminant of real symmetric matrices. Commun. Pure Appl. Math. 51(11-12), $1387-1396(1998)$

28. Lazard, D.: On the representation of rigid-body motions and its application to generalized platform manipulators. In: Angeles, J., Hommel, G., Kovàcs, P. (eds.) Computational Kinematics. Solid Mechanics and Its Applications, vol. 28, pp. 175-182. Kluwer, Dordrecht (1993)

29. Lazard, D.: On the specification for solvers of polynomial systems. In: 5th Asian Symposium on Computers Mathematics-ASCM 2001. Lecture Notes Series in Computing, vol. 9, pp. 66-75. World Scientific, Singapore (2001)

30. Levin, J.: A parametric algorithm for drawing pictures of solid objects composed of quadric surfaces. Commun. ACM 19(10), 555-563 (1976)

31. Maple System: Waterloo Maple Software. http://www.maplesoft.com

32. Milenkovic, V.J.: Robust construction of the Voronoi diagram of a polyhedron. In: Proceedings of the 5th Canadian Conference on Computational Geometry (CCCG'93), pp. 473-478 (1993)

33. Mourrain, B., Técourt, J.-P., Teillaud, M.: On the computation of an arrangement of quadrics in 3D. Comput. Geom. Theory Appl. 30(2), 145-164 (2005) (Special issue, 19th European Workshop on Computational Geometry)

34. Mulzer, W., Rote, G.: Minimum-weight triangulation is NP-hard. J. ACM 55(2), 1-29 (2008)

35. Okabe, A., Boots, B., Sugihara, K., Chiu, S.N.: Spatial Tessellations-Concepts and Applications of Voronoi Diagrams, 2nd edn. Wiley, New York (2000)

36. Parillo, P., Papachristodoulou, A., Prajna, S., Seiler, P.: SOSTOOLS-A MATLAB toolbox for sums of squares optimization programs. http://www.cds.caltech.edu/sostools/

37. Robertson, N., Sanders, D.P., Seymour, P.D., Thomas, R.: The four colour theorem. J. Comb. Theory Ser. B 70, 2-44 (1997)

38. Rouillier, F., Roy, M.-F., Safey El Din, M.: Finding at least one point in each connected component of a real algebraic set defined by a single equation. J. Complex. 16, 716-750 (2000)

39. Safey El Din, M.: Testing sign conditions on a multivariate polynomial and applications. Math. Comput. Sci. 1(1), 177-207 (2007)

40. Safey El Din, M.: RAG'Lib-A library for real algebraic geometry. http://www-calfor.lip6.fr/ safey/ RAGLib/

41. Safey El Din, M., Schost, E.: Polar varieties and computation of at least one point in each connected component of a smooth real algebraic set. In: Proceedings of the International Symposium on Symbolic and Algebraic Computation (ISSAC'03), pp. 224-231. ACM, Philadelphia (2003)

42. Safey El Din, M., Schost, E.: Properness defects of projections and computation of one point in each connected component of a real algebraic set. Discrete Comput. Geom. 32(3), 417-430 (2004)

43. Schömer, E., Wolpert, N.: An exact and efficient approach for computing a cell in an arrangement of quadrics. Comput. Geom. Theory Appl. 33(1-2), 65-97 (2006) (Special Issue on Robust Geometric Algorithms and Their Implementations)

44. Schwartz, J.T., Sharir, M.: On the "piano movers" problem: V. The case of a rod moving in threedimensional space amidst polyhedral obstacles. Commun. Pure Appl. Math. 37, 815-848 (1984)

45. Schwarzkopf, O., Sharir, M.: Vertical decomposition of a single cell in a three-dimensional arrangement of surfaces and its applications. Discrete Comput. Geom. 18, 269-288 (1997)

46. Segre, C.: Studio sulle quadriche in uno spazio lineare ad un numero qualunque di dimensioni. Mem. della R. Acc. delle Scienze di Torino 36(2), 3-86 (1883)

47. Sharir, M.: Almost tight upper bounds for lower envelopes in higher dimensions. Discrete Comput. Geom. 12, 327-345 (1994)

48. Teichmann, M., Teller, S.: Polygonal approximation of Voronoi diagrams of a set of triangles in three dimensions. Technical report 766, Laboratory of Computer Science, MIT (1997)

49. Viro (Drobotukhina), J., Viro, O.: Configurations of skew lines. Leningrad Math. J. 1(4), 1027-1050 (1990) (Revised version in English: arXiv:math/0611374) 